\title{
1 Assembly of functionally antagonistic visual circuits for controlling pupil dynamics
}

3 Onkar S. Dhande ${ }^{1, *}$, Tania A. Seabrook ${ }^{1}$, Ann H Phan ${ }^{1}$, Lindsey D. Salaly ${ }^{1}$, Nao Ishiko ${ }^{1}$,

4 Phong L. Nguyen ${ }^{1, \dagger}$, Jack T. Wang ${ }^{1}$, Sylvia M. Evans ${ }^{2}$, Andrew D. Huberman ${ }^{1,3,4,5, \text { * }}$

6 1. Department of Neurobiology, Stanford University School of Medicine, Stanford, CA

794305, USA

8 2. Department of Medicine, University of California, San Diego, 9500 Gilman Drive, La

9 Jolla, CA 92093, USA

10 3. Department of Ophthalmology,

11 4. Stanford Neurosciences Institute, and

12 5. BioX, Stanford University School of Medicine, Stanford, CA 94305, USA

14 tCurrent address: Salk Institute for Biological Studies, La Jolla, CA

16 *Corresponding authors: Onkar Dhande odhande@stanford.edu and Andrew Huberman

17 adh1@stanford.edu

18

19 Lead Contact: Andrew Huberman adh1@ stanford.edu 


\section{$32 \quad$ Highlights}

33

34 - Tbx20 plays a role in the development of non-image-forming pathways.

- Loss of Tbx20-expressing RGCs alters specific aspects of the pupil reflex arc.

- Previously unknown role for a specific subset of Tbx20-expressing RGCs in modulating pupil size.

\section{1 eTOC}

42 Dhande et al. identify a novel genetic program that marks and is required for the

43 development of non-image-forming parallel visual pathways. Moreover, chemogenetic

44 activation of a specific retina-to-OPN pathway reveals a novel circuit element controlling

45 pupil size. These findings provide new insight into the mechanisms that establish defining

46 features of functionally specialized sensory pathways.

47 


\section{Summary}

49

50 The functional attributes of parallel sensory circuits are determined by the properties of the

51 individual cell types that comprise them, and their connectivity. The mechanisms

52 controlling the specification and establishment of parallel sensory pathways remain

53 unclear. Here we report the non-uniform distribution of retinal ganglion cells (RGCs)

54 expressing the transcription factor Tbx20 in mice. Owing to their layout within the retina,

55 Tbx20-RGCs are positioned to preferentially encode light information from dorsal visual

56 space. We discovered that Tbx20 expression is mostly restricted to three RGC-types: M1

57 intrinsically photosensitive RGCs (ipRGCs), M2 ipRGCs and 'diving' RGCs. The axonal

58 projections of Tbx20-RGCs innervate non-image-forming brain centers including the

59 suprachiasmatic nucleus, the medial division of the posterior pretectal nucleus, and the

60 olivary pretectal nucleus (OPN), a principal station in the neural pathway for generating

61 the pupillary light reflex (PLR). Conditional deletion of Tbx20 resulted in reduced Tbx20-

62 RGC axonal innervation of these targets and revealed a key role of these neurons in driving

63 specific phases of the PLR. Furthermore, chemogenetic activation uncovered a novel role

64 for a specific subset of Tbx20-RGCs in controlling pupil dilation. These data offer a new

65 understanding of the genetic and molecular mechanisms that establish specific,

66 behaviorally-relevant visual circuits.

67

68 Keywords: Retinal ganglion cells; subcortical; pretectum; pupillary light reflex; olivary

69 pretectal nucleus; ipRGC; Tbx20; target specificity; mouse vision; chemogenetics 
80

81

82

83

84

85

86

\section{Introduction}

A hallmark feature of the mammalian eye-to-brain pathway is that the cells and circuits carrying specific aspects of visual information are often anatomically segregated from one another into parallel pathways. In the last decade, genetic tools have played a pivotal role in elucidating the identity of the specific retinal output neurons, the retinal ganglion cells (RGCs), and the targets they connect to in the brain (Hattar et al., 2002, Huberman et al., 2008; 2009; Kim et al., 2010; Kay et al., 2011; Dhande et al., 2013; 2015). What remains fundamentally lacking, however, is an understanding of how individual feature-detection channels within those parallel pathways are specified and established during development and how the RGCs within each discrete channel contribute to the operation of the circuit and consequently, how they drive behavioral outputs.

A prime example of a functionally dedicated pathway is the non-image-forming pathway serving the pupillary light reflex (PLR). The PLR allows the visual system to titrate the amount of light reaching the photoreceptors, reduce optical aberrations, and increase the effective focus, thereby optimizing visual sensitivity and acuity (Denton 1956; Campbell \& Gregory, 1960). The primary stages of the PLR involve eye-to-brain signaling from a dedicated set of RGCs that relay changes in overall ambient light levels reaching the eye, to neurons in the OPN - a bilaterally symmetric set of targets in the dorsal midbrain (Trejo \& Cicerone 1984; Young and Lund 1994; Gamlin and Clarke 1995; Gamlin et al. 1995; Distler \& Hoffmann 1989a, b).

A specific category of retinal projection neurons termed 'intrinsically photosensitive RGCs' (ipRGCs) is crucial for the integrity of the PLR. The ipRGCs are sonamed because they not only encode light signals from rod/cone photoreceptors but also express the photopigment melanopsin/OPN4 and thus are endowed with the property of responding directly to light (i.e. melanopsin-dependent depolarization). As a group, ipRGCs include five subtypes, 'M1-M5', each with different morphologies, degrees of photosensitivity, patterns of central connections and thus functional roles (Berson et al., 2002; Hattar et al., 2002; Sand et al., 2012). Previous work demonstrated that the axons from different ipRGC subtypes are compartmentalized within the OPN. The M1 ipRGCs express the highest levels of melanopsin and thus display the strongest intrinsic photosensitivity and project their axons to the 'shell' of the OPN, whereas the M2 ipRGCs 
112 and the 'diving' RGCs project to the OPN 'core' (Fig. 1A-A', Schematized in Fig. 1B)

113 (Hattar et al., 2002, 2006; Baver et al., 2008; Osterhout et al., 2011; 2014). The shell-

114 projecting M1 ipRGCs are necessary for generating a normal light-driven PLR in response

115 to changes in environmental light levels; ablating these M1s drastically attenuates the PLR

116 (Güler et al., 2008). Given that M1s project to the OPN shell, this raises the question: what

117 is the functional relevance of retino-OPN core connectivity? Indeed, the functional role of

118 the OPN core remains unknown and the specific output channels of the shell versus core

119 remain mysterious, as does their greater role in driving the PLR and other behaviors.

120 Here we used molecular genetic tools centered around the T-box transcription

121 factor Tbx20 to visualize a specific subset of non-image-forming RGCs including those

122 that project from the eye to the 'core' compartment of the OPN, along with cell-type-

123 specific profiling, conditional knockout of Tbx20 and in vivo behavioral analyses, to parse

124 the organizational logic and mechanisms underlying the specification of non-image

125 forming visual circuits. We discovered a molecularly defined subset of RGCs that are

126 arranged in a unique distribution across the retinal surface and project to distinct target

127 regions within the non-image-forming pathway. We then used conditional alleles of Tbx20

128 and chemogenetic activation to discover that Tbx20 plays a crucial role in establishing 129 specific non-image-forming pathways which we find modulate pupil size. These findings

130 are discussed in terms of the potential role of this circuit in visual behaviors and in the

131 context of understanding how parallel sensory pathways are designated and assembled.

\section{Results}

Cdh3-RGCs are non-uniformly distributed in the retina

136 In the transgenic mouse line Cdh3-GFP, the retina expresses enhanced GFP (GFP) in a 137 subset of M2-type ipRGCs and 'diving' RGCs (hereafter referred to as Cdh3-RGCs), which

138 project their axons to a specific set of non-image-forming centers in the brain: the OPN139 core, medial division of the posterior pretectal nucleus (mdPPN) and ventral lateral 140 geniculate nucleus (vLGN) (Osterhout et al., 2011; 2014). The spatial distribution of 141 specific RGC-types across the retina has direct implications for the kind of visual 142 information processed by their respective target in the brain. Therefore, we systematically 143 examined the distribution of Cdh3-RGCs $\left(\mathrm{GFP}^{+}\right)$and observed they are arranged in a 
144 striking ventral:high, dorsal:low density-distribution across the retinal surface (Fig. 1C).

145 The distribution of Cdh3-RGCs was significantly biased towards the ventral half of the

146 retina $(\mathrm{p}<0.001$, Fig. 1D left). However, no significant bias was observed for the

147 distribution of Cdh3-RGCs between the nasal and temporal half of the retina $(\mathrm{p}=0.2$; Fig.

148 1D right). This ventrally-biased layout indicates that Cdh3-RGCs are poised to encode

149 visual features preferentially from the dorsal visual field. Furthermore, this also implies

150 that target nuclei receiving input from these RGCs are positioned to extract visual

151 information from the dorsal visual field. Together, the characteristics of Cdh3-RGCs and

152 their central projections to the midbrain reveal a unique subdivision of the retinofugal

153 pathway.

155 Tbx20, a T-box family transcription factor, is highly enriched in Cdh3-RGCs

156 The identification of molecular markers for M1 ipRGCs has greatly facilitated the study

157 and understanding of their development and function (McNeill et al., 2011; Chen et al.,

158 2011; Fox \& Guido, 2011). Similarly, to further understanding of the retino-OPN pathway

159 we performed an immunohistochemical screen to identify molecular markers for Cdh3-

160 RGCs, focusing specifically on transcription factors. Those experiments revealed Tbx20,

161 a T-box family member, as an intriguing candidate marker for Cdh3-RGCs.

162 To determine the expression of Tbx20 in Cdh3-RGCs, we stained whole mount

163 retinas from Cdh3-GFP mice with an antibody specific to Tbx20 protein (Song et al., 2006).

164 More than $80 \%$ of Cdh3-RGCs expressed Tbx20 at postnatal day 4 (P4) (Fig. 1E-E", 1F).

165 Conversely, Cdh3-RGCs comprised a smaller fraction ( 38\%) of the Tbx20 expressing

166 population of retinal neurons (Fig. 1F). To determine the other types of RGCs that express

167 Tbx20 and their distributions across the retina, we also used a GFP mouse line expressing

168 a Tbx20-GFP fusion protein, which recapitulates the endogenous nuclear expression of

169 Tbx20 (Shen et al., 2011). First, examination of the spatial arrangement of Tbx20-RGCs

170 (Tbx20-GFP-expressing) revealed a ventral: high, dorsal: low gradient of Tbx20-RGC

171 soma density (Fig. 1G, H, and Fig. S1). The biased distribution of Tbx20-RGCs was

172 already apparent in flat-mounted retinas on embryonic day (E) 18.5 (Fig. S1A-A2), a

173 developmental time point when most RGCs have differentiated (Young 1985) and persisted

174 through the early postnatal weeks and into adulthood (Fig. S1B-D). We found the 
175 distribution of Tbx20-RGCs at P20 was significantly biased towards the ventral half of the 176 retina, similar to $\mathrm{Cdh} 3-\mathrm{RGCs}(\mathrm{p}=0.0016$, Fig. $1 \mathrm{H}$ left). Although there was a strong

177 temporal bias in the distribution of Tbx20-RGCs in 4/5 retinas, on average the distribution

178 of Tbx20-RGs in temporal versus nasal retina was not significantly different $(\mathrm{p}=0.2$, Fig.

179 1H right). Thus, both Tbx20-RGCs and Cdh3-RGCs have a similar dorsal:low to

180 ventral:high gradient, although Tbx20-RGCs have a qualitative bias towards the ventral-

181 temporal quadrant (compare Fig. 1G, H to Fig. 1C, D). These data suggest that the non-

182 uniform distribution of Tbx20-RGCs is an early and persistent feature of this cell type, and

183 not the transient consequence of postnatal events that shape retinal circuitry (e.g., naturally

184 occurring developmental cell-death) (Young 1984; Crespo et al., 1985; Williams et al., 185 1986).

186 Second, by staining retinas from Tbx20-GFP mice for other markers of RGC 187 identity, we found that almost all Tbx20-RGCs express Tbr2 ( 96\%; Fig. S2A-A”, 2F), 188 which is a marker of non-image-forming RGCs (Mao et al., 2014; Sweeney et al., 2014, 189 2017; Seabrook et al., 2017). Additionally, we found that many Tbx20-RGCs express 190 Brn3b (Mean \pm SEM: $76 \pm 6 \%, n=4$ mice/retinas, 465 Tbx20-GFP cells; data not shown), 191 a transcription factor known to play a role in the development of non-image-forming 192 RGCs, including ipRGCs, and their central projections (Quina et al. 2005; Badea et al., 193 2009). A negligible number of Tbx20-RGCs express Cart ( 1\%), a marker of On-Off 194 direction-selective RGCs (Kay et al., 2011), or SMI32 ( 1\%), a marker of alpha-like RGCs

195 (Lim et al., 2007) (Fig. S2B-C', 2F). A small fraction of Tbx20-RGCs ( 10\%) express the 196 photopigment melanopsin at high enough levels to be detected by standard 197 immunohistochemistry (Fig. S2D-D”, 2F). Together, these data suggest that Tbx20 is 198 expressed by a mixed but relatively restricted subset of non-image-forming RGCs, 199 including those projecting to the OPN core.

201 Tbx20 loss results in reduced retinal innervation of non-image-forming targets

202 Does Tbx20 play a role in establishing non-image-forming pathways? To address this, we 203 used the Cre-loxP system to genetically delete Tbx20 from non-image-forming RGCs. We 204 screened the GENSAT repository of BAC transgenic mice (Gong et al., 2003) to identify 205 lines in which Cre recombinase was expressed in non-image-forming RGCs. From that 
206 screen, we identified the Tph2-Cre mouse line $\left(\mathrm{Cre}^{+}\right)$as a potential candidate. We found 207 that $\sim 95 \%$ of Tbx20-RGCs express Cre recombinase in the Tph2-Cre mouse line (Fig. 2A208 B). Moreover, we previously reported that in the Tph2-Cre mouse line the majority of non209 image-forming RGCs express Cre [Fig. 2C-C"; previously quantified: $84 \%$ of melanopsin ${ }^{+}$ 210 ipRGCs, 82\% of Tbr2+ RGCs, and 90\% of Cdh3-RGCs express Tph2-Cre (Seabrook et al., 211 2017)]. Thus, we crossed $\mathrm{Cre}^{+}$mice to transgenic mice harboring floxed alleles of Tbx20 $212\left(\mathrm{Tbx} 20^{\mathrm{f} / \mathrm{f}}\right)$ in order to generate heterozygous Tbx20 conditional knockout (cKO) offspring $213\left(\mathrm{Cre}^{+} ; \mathrm{Tbx} 20^{\mathrm{f} /+}\right)$. These mice were in turn crossed to $\mathrm{Tbx} 20^{\mathrm{f} / \mathrm{f}}$ to produce $\mathrm{Tbx} 20$ conditional 214 knockout mice (Cre ${ }^{+}$; Tbx20 $0^{\mathrm{f} / \mathrm{f}}$ ) (hereafter referred to as 'Tbx20 cKO mice' for simplicity).

215 To assess the consequence of deleting Tbx20 on the non-image-forming pathways 216 we first visualized the entire retinofugal pathway in control and Tbx20 cKO mice by 217 injecting the anterograde fluorescent tracer CT $\beta-488$ into both eyes to label RGC axons. 218 The OPN, a major target of Cdh3-RGCs and M1 ipRGCs, and the suprachiasmatic nucleus 219 (SCN), a major target of M1 ipRGCs, were clearly identifiable based on CT $\beta^{+} \mathrm{RGC}$ axonal 220 labeling in Tbx20 cKO mice (Fig. 2D-G). The OPN and SCN area occupied by CT $\beta^{+}$RGC 221 axons was not significantly different between control and Tbx20 cKO mice (data not 222 shown). However, the innervation density of RGC axons in the OPN (core and shell) and 223 the SCN was significantly reduced in Tbx20 cKO mice, in comparison to control mice 224 (OPN: $\sim 28 \%$ reduction, $\mathrm{p}=0.008 ; \mathrm{SCN}: \sim 58 \%$ reduction, $\mathrm{p}=0.019 ;$ Fig. $2 \mathrm{H}, \mathrm{I})$. These data 225 suggest that the loss of Tbx20 function leads to a disruption of the parallel pathways 226 forming the non-image-forming system.

\section{A role for $\mathbf{T b x 2 0}$ in establishing the retino-OPN-core parallel pathway}

229 What is the consequence of deleting Tbx20 on OPN-core-projecting RGCs? To visualize 230 this we generated Tbx20 cKO mice on a Cdh3-GFP background, yielding Tph2-Cre ${ }^{+}$; $231 \mathrm{Cdh} 3-\mathrm{GFP}^{+} ; \mathrm{Tbx} 20^{\mathrm{f} / \mathrm{f}}$ triple transgenic/conditional mutant mice. By examining the axonal 232 projections of Cdh3-RGCs in the brains of juvenile Tbx20 cKO mice and in littermate233 control mice (Tph2-Cre ${ }^{+}$Cdh3-GFP ${ }^{+}$; Tbx20 $0^{\mathrm{f} /+}$, and Cdh3-GFP ${ }^{+} ; \mathrm{Tbx} 20^{\mathrm{f} / \mathrm{f}}$, and Cdh3$234 \mathrm{GFP}^{+}$; Tbx20 ${ }^{\mathrm{f} /+}$; P19-P25) we found that, in the absence of Tbx20 function, Cdh3-RGC 235 innervation of the OPN-core was substantially reduced. In 2/7 Tbx20 cKO mice, the 236 projections were modestly reduced (Fig. 3Q, 3R) and in 5/7 mice, the projections to this 
237 target were completely absent (Fig. 3B-E, 4P, schematized in Fig. 3Y). The same general 238 effect was observed for the mdPPN, a target situated immediately caudal to the OPN. 239 Normally, the mdPPN is densely innervated by Cdh3-RGC axons (Fig. 3F), but after 240 conditional removal of Tbx20 from Cdh3-RGCs, the mdPPN was also largely devoid of 241 Cdh3-RGC axonal innervation (Fig. 3G-J, 3S-U, schematized in Fig. 3Y). Cdh3-RGC 242 inputs to the vLGN- another brain target that normally receives substantial input from 243 Cdh3RGCs, were absent or substantially reduced in Tbx20 cKO mice (Fig. 3L-O, 3V-X). 244 Is the lack of target innervation by Cdh3-RGCs observed in Tbx20 cKO mice due 245 to a loss of Cdh3-RGCs? To address this, we first quantified the number of Cdh3-RGCs 246 present in Tbx20 cKO retinas (see methods). We found that only a small number of Cdh3247 RGCs remained in Tbx20 cKO mice (Mean \pm SEM: $30 \pm 8$, n=7 mice/retinas; Fig. 3Z).

248 This indicates that conditional deletion of Tbx20 results in a severe reduction in Cdh3249 RGCs. Moreover, $>90 \%$ of the spared Cdh3-RGCs in Tbx20 cKO retinas did not express 250 Cre [confirmed by the absence of tdTomato (Cre-dependent reporter); Fig. 3Z] and thus, 251 any Cdh3-RGCs spared was the consequence of lack of Cre dependent Tbx20 excision, not 252 a failure for Tbx20 deletion to eliminate these cells. The absence of Cdh3-RGC cells in 253 Tbx20 cKO retinas suggests that the reduction in Cdh3-RGC inputs to the OPN-core 254 observed in Tbx20 cKO mice reflects the loss of Cdh3-RGCs altogether. However, these 255 data cannot exclude the possibility that loss of Tbx20 might somehow result in a loss 256 of GFP expression in Tbx20 cKO mice.

\section{Loss of Tbx20 partially disrupts the organization of the retino-OPN-shell pathway}

259 It is well established that M1 ipRGCs express the highest levels of the photopigment 260 melanopsin relative to all other ipRGC types and as a result, display the greatest intrinsic 261 light responses (Schmidt et al., 2011; Sand et al., 2012). Furthermore, M1 ipRGCs are the 262 exclusive source of retinal inputs to the OPN shell- the pathway thought to drive the PLR 263 (Hattar et al., 2002; Viney et al., 2007; Baver et al., 2008; Berson et al., 2010; Güler et al., 264 2008). A small fraction of Tbx20-RGCs express high levels of melanopsin (Fig. 1I, Fig. 265 S2D-D'); therefore it seemed reasonable to assume that some of those Tbx20-RGCs might 266 be M1 ipRGCs. Hence, we sought to determine if Tbx20 function is necessary for normal 267 development of the retino-OPN-shell pathway. 
As a first step toward that goal, we stained flat-mounted retinas from Tbx20 cKO mice and littermate-control mice with an antibody against melanopsin to visualize M1 ipRGCs. The somas and dendrites of M1 ipRGCs show the strongest immunoreactivity for melanopsin (Berson et al., 2010; see Methods for details on antibody specificity). In Tbx20 cKO mice, the layout of the network of ipRGC types was severely disrupted, although some M1 ipRGCs were still present (Fig. 4A-B', schematized in Fig. 4C). The severe but

274 incomplete loss of M1 ipRGCs is consistent with our finding that not all ipRGCs that 275 express high levels of melanopsin also express Tbx20 protein (see above).

To more directly explore the consequence of Tbx20 loss on the organization of the 277 OPN-shell projections we utilized a transgenic mouse line in which a membrane-targeted 278 reporter (Tau-LacZ) was knocked into the Opn4 (melanopsin) locus (Opn4 ${ }^{\text {TauLacZ; Hattar et }}$ al., 2002, 2006). This reporter line allows for the visualization of M1 ipRGCs and their central projections, including those to the OPN shell. We generated Tbx20 cKO mice on as described above. The significantly lower density of $\beta$-gal staining in the OPN of these mice also suggests a reduction in the innervation of the OPN $(\mathrm{p}=0.02$; Fig. $5 \mathrm{~A}-\mathrm{H}, 5 \mathrm{O}$, schematized in Fig. 5Q). Also, the organization of the M1 ipRGC inputs to the OPN shell was more diffuse in comparison to littermate-control mice (Fig. 5A-H). Furthermore, we found that M1 ipRGC innervation of the SCN, the other major target of these RGCs, was significantly reduced ( $\mathrm{p}<0.001$; Fig. 5I-N, 5P). Thus, Tbx20 cKOs have both a loss of Cdh3-RGC inputs to the OPN-core and partial loss of M1 ipRGC inputs to the OPN-shell.

\section{Loss of Tbx20 function does not alter the development of other parallel pathways}

291 The Tph2-Cre mouse line $\left(\mathrm{Cre}^{+}\right)$is a highly useful tool for genetically manipulating Tbx20-

292 RGCs. However, a small fraction of other RGC subtypes (non-Tbx20 expressing) also 293 express Cre recombinase in this mouse line: <3\% of image-stabilizing RGCs [Hoxd10294 RGCs (Dhande et al., 2013); Seabrook et al., 2017], 65\% of Cart-expressing RGCs 295 (includes On-Off direction-selective RGCs, Fig. S4A) and 37\% of alpha-like RGCs (Fig. 296 S4A) express Cre recombinase. Does deletion of Tbx20 impact the development of these 297 optic pathways? We examined the axonal projections of Hoxd10-RGCs in the brains of 298 juvenile (P26) Tbx20 cKO mice (Tph2-Cre ${ }^{+}$; Hoxd10-GFP ${ }^{+}$Tbx20/f) and littermate- 
299 control mice (Tph2-Cre ${ }^{+}$; Hoxd10-GFP ${ }^{+}$; Tbx20 $0^{\mathrm{f} /+}$, and Hoxd10-GFP+; Tbx20 $0^{\mathrm{f} / \mathrm{f}}$, and 300 Hoxd10-GFP ${ }^{+} ; \mathrm{Tbx}_{20}^{\mathrm{f} /+}$ ) and found that, in the absence of Tbx20 function, Hoxd10-RGCs

301 still innervate their correct targets (Fig. S3A-F). Furthermore, the density of target 302 innervation by the axons of Hoxd10-RGCs mice is not significantly different between 303 Tbx20 cKO mice and control mice (NOT: $\mathrm{p}=0.64$; MTN: $\mathrm{p}=0.56$; DTN: $\mathrm{p}=0.34$, Fig. S3G-

304 I). Next, we stained Tbx20 cKO and control retinas for RGC subtype markers, Cart and 305 SMI32 and found that loss of Tbx20 does not alter the density of either RGC subtype306 specific marker (Fig. S4B-G). These data suggest that Tbx20 is required specifically for 307 the development of the non-image-forming visual pathways.

309 Loss of Tbx20-RGCs minimally impacts PLR

310 What is the contribution of Tbx20-RGCs to the PLR? The consensual PLR is defined as 311 the constriction of one eye's pupil caused by light-stimulating the opposite eye; an effect 312 thought to be mediated by bilateral projections from the OPN to downstream brainstem 313 circuits (Young and Lund, 1994). The ipRGC inputs to the OPN are essential for driving 314 this behavior (Güler et al., 2008; Hatori et al., 2008; Badea et al., 2009; Chen et al., 2011; 315 Sweeney et al., 2014; Mao et al., 2014; Seabrook et al., 2017).

316 Given the retino-OPN pathway is disrupted in Tbx20 cKO mice, we wanted to 317 know if and how Tbx20-RGCs exert a role in the generation and/or modulation of the 318 consensual pupillary response. Dark-adapted mice were manually restrained and the 319 change in the pupil size of one eye was continuously recorded in response to stimulation 320 of the other eye with a brief (30s) pulse of light. The relative pupil area and percent 321 constriction/dilation were quantified from images extracted from the recording at $5 \mathrm{~s}$ 322 intervals (see Methods for details). The consensual PLR in Tbx20 cKO mice and littermate 323 controls was tested using a blue LED $(470 \mathrm{~nm})$, a wavelength that efficiently stimulates 324 ipRGCs and drives robust PLR responses (Lucas et al., 2001, 2003; Berson et al., 2002; 325 Gamlin et al., 2007). In addition, because the PLR functions over a range of luminance 326 levels, we analyzed consensual PLR at multiple light levels (100 lux, 1,000 lux and 10,000 $327 \operatorname{lux})$.

328 First, we computed the average pupil constriction (\%) at these three light levels and 329 found that, at 100 lux, the PLR was significantly reduced but the effect was one of only 
330 mild attenuation compared to littermate controls ( 7\% less constricted, $\mathrm{p}=0.045$; Fig. 6A', 331 6A"). The same general effect of a significant (but still mild) reduction compared to 332 controls was also observed at 1,000 lux ( 10\% less constricted, p=0.0014; Fig. 6B', 6B”). 333 Pupil constriction was not significantly different between mice lacking Tbx20-RGCs and 334 control mice at 10,000 lux ( 3\% less constricted, p=0.06; Fig. 6C', 6C"). These data 335 indicate that Tbx20-RGCs are mostly dispensable for generating a PLR response. Tbx20336 RGCs appear to influence PLR to a small extent only at relatively low light levels (100 lux 337 and 1,000 lux).

338 OPN-shell projecting M1 ipRGCs are required for generating light-induced pupil 339 constriction (Güler et al, 2008). Given the small but significant decrease in PLR response 340 in Tbx20 cKO mice we wondered whether the near normal PLR response in Tbx20 cKO 341 was driven by the remaining OPN-shell projecting M1 ipRGCs in Tbx20 cKO mice (see 342 above). To dampen the drive of the residual OPN-shell projecting M1 ipRGCs, we 343 generated Tbx20 cKO mice that also lacked the photopigment melanopsin (Mel KO: Opn4 344 TauLacZ/TauLacZ; see Methods) - hereafter referred to as Tbx20/Mel dKO mice (Tph2-Cre ${ }^{+}$; 345 Tbx20 f/ff; Opn4 TauLacZ/TauLacZ, dKO: double knockout mice). Previous work showed that 346 eliminating melanopsin-photopigment signaling results in a loss of intrinsic 347 photosensitivity in M1 ipRGCs, which in turn leads to reduced pupil constriction in 348 response to light increments (Lucas et al., 2003, Hattar et al., 2003; Panda et al., 2003; 349 Jagannath et al., 2015; Mure et al., 2016). We then tested the consensual PLR in Tbx20/Mel $350 \mathrm{dKO}$ mice. At all three light levels: 100 lux, 1,000 lux and 10,000 lux, pupillary 351 constriction in $\mathrm{Tbx} 20 / \mathrm{Mel} \mathrm{dKO}$ mice was dramatically reduced relative to mice lacking 352 Tbx20 alone $(\sim 34 \%, \sim 37 \%$, and $\sim 32 \%, \mathrm{p}<0.0001$; less constriction respectively; Fig. 6A', 353 6B", 6B', 6B”, 6C'and 6C'). Similarly, the PLR of Tbx20/Mel dKO mice was drastically 354 reduced relative to control mice at all three light levels (100 lux: 41\%, 1,000 lux: 45\%, 355 10,000 lux: 38\% less constricted, p<0.0001; Fig. 6A', 6A”, 6B', 6B”, 6C', 6C”).

356 As discussed above, the PLR is disrupted in mice lacking melanopsin signaling 357 alone. Therefore, we examined whether the severe attenuation in PLR in Tbx20/Mel dKO 358 mice predominantly reflects the loss of melanopsin signaling. The average pupillary 359 constriction (\%) in Mel KO mice was significantly less than control mice at 100 lux ( 13\% 360 less constricted, p<0.001; Fig. 6A', 6A”). Similarly, the PLR was significantly attenuated 
361 compared to controls at 1,000 lux and 10,000 lux ( 8\% less constricted, $\mathrm{p}=0.0023$; and $362 \sim 13 \%$ less constricted, p<0.0001; respectively; Fig. 6B', 6B”, 6C, 6C”). Thus, Mel KOs 363 display a significant, although minor, attenuation in PLR at all light levels, a trend similar 364 to that observed in Tbx20 cKOs. Taken together these data indicate that, in Tbx20 cKOs, 365 visual drive provided by the remaining M1 ipRGCs is sufficient for generating PLR. 366 Furthermore, the severity of the PLR loss in Tbx20/Mel double KO mice implies that 367 Tbx20-RGCs cooperatively acts with the OPN-shell pathway to drive the PLR.

368 Finally, we tested whether the PLR deficits in the mutant mice were somehow a 369 result of abnormal function of the ciliary muscles used for constricting the pupil. 370 Application of the carbachol, a muscarinic agonist that activates the ciliary muscles 371 (Maayani et al., 1975), caused complete pupillary constriction in both control and mutant 372 animals, indicating that ciliary muscle function is unaffected in mutant mice (data not 373 shown).

Melanopsin- and photoreceptor-mediated-signaling cooperatively act to generate the post-illumination PLR

In addition to studying the pupil constriction that occurs after the onset of a light stimulus, 379 is the sustained pupil constriction after the offset (>30s) of a high intensity, blue light 380 stimulus (Gamlin et al., 2007). In humans and macaque monkeys, the PIPR is primarily 381 mediated by melanopsin signaling (Gamlin et al., 2007; Mure et al., 2009). Sustained 382 depolarization of ipRGCs after the offset of light, due to the slow deactivation of 383 melanopsin, is thought to generate the PIPR (Gamlin et al., 2007). Indeed, a recent report 384 showed that in the presence of a truncated version of melanopsin (lacking residues beyond 385 amino acid 397) that deactivates faster than normal melanopsin, mice fail to maintain 386 pupillary constriction after light offset (Mure et al., 2016).

Following transient illumination of the eye with relatively low-intensity light (100 388 lux or 1,000 lux) the pupil constricts and then dilates to return to baseline levels (i.e., same 389 as dark adapted levels). This was observed for all the genotypes tested (Controls, Tbx20 $390 \mathrm{cKO}$, Mel KO, and Tbx20/Mel dKO) (Fig. 6A-6B','). However, after transient exposure 391 of the eye to light of a relatively high luminance (10,000 lux) the PIPR occurs at a stage 
392 30-60s after light offset. Similar to the PLR, the disruption in PIPR in Tbx20 cKO mice 393 was modest and not significantly different from controls ( $5 \%$ more dilated, $p=0.44$, Fig.

$3946 C^{\prime \prime \prime)}$ ). If melanopsin-signaling alone underlies PIPR, then we would expect Mel KO mice 395 to reach baseline levels of pupil dilation after the offset of an intense light stimulus. 396 Surprisingly, after intense light offset, although the pupils of Mel KOs did dilate 397 significantly more than the pupils of Tbx20 cKOs $(\sim 25 \%$ more dilated, p<0.0001; Fig. $3986 \mathrm{C}^{\prime \prime \prime}$ ) the dilation of Mel KO pupils did not reach baseline levels (i.e., complete dilation).

399 These data suggest that, in addition to melanopsin-signaling, other mechanisms influence 400 the PIPR. In the absence of melanopsin-signaling the only source of visual information that 401 can drive any aspect of the PLR or PIPR are light signals encoded by conventional rod/cone 402 photoreceptors relayed to the brain via OPN-projecting RGCs. Indeed, when we eliminated 403 melanopsin signaling and genetically ablated Tbx20-RGCs (Tbx20/Mel dKOs), we found 404 that, on average, the pupils dilate to near baseline levels ( $85 \%$ dilation) between 30-60s 405 after the offset of the light stimulus ( $51 \%$ more dilated than Tbx20 cKO, p $<0.0001$; Fig. 406 6C'"). Together, these data suggest that not only are Tbx20-RGCs important for the PIPR, 407 but that conventional photoreceptor responses play a significant role in generating the 408 PIPR.

\section{Targeting Cdh3-RGC using the Cre-DOG system}

411 The loss of both Tbx20-RGC projections to the OPN-core and to the OPN-shell in Tbx20 412 cKO mice confounds the dissociation of the role of OPN-core versus OPN-shell projecting 413 RGCs in generating PLR. Therefore, to more directly test a role of OPN-core projecting 414 Cdh3-RGCs in the PLR we used a chemogenetic tool hM3Dq $\left[\mathrm{G}_{\mathrm{q}}\right.$-coupled human 415 muscarinic $\mathrm{M}_{3}(\mathrm{hM} 3 \mathrm{Dq})$ designer receptor exclusively activated by designer drugs 416 (DREADDs)]. hM3Dq is an excitatory DREADD that dramatically increases neuronal 417 firing when bound by its synthetic ligand Clozapine N-oxide (CNO) (Urban and Roth, 418 2015, schematized in Fig. S5A). Previously, our lab demonstrated that hM3Dq-expressing 419 RGCs increased their spontaneous and light-evoked firing when exposed to CNO (Lim et 420 al., 2016).

421 To modulate the activity of Cdh3-RGCs, we took advantage of the 'floxed-Stop422 hM3D' transgenic mouse line that expresses hM3Dq in a Cre-dependent manner (Zhu et 
423 al., 2016). To test the efficiency of chemogenetic activation of RGCs in this mouse line,

424 we injected adeno-associated viruses (AAVs) encoding Cre recombinase into the eyes of 425 floxed-Stop-hM3D $\left(\mathrm{Cre}^{+} / \mathrm{hM}^{+}\right)$mice to achieve widespread expression of $\mathrm{hM} 3 \mathrm{D}$ in a 426 random population of RGCs (Fig. S5B, C; n=2 mice). In the absence of CNO, the dark427 adapted pupils of $\mathrm{Cre}^{+} / \mathrm{hM} \mathrm{D}^{+}$mice were completely dilated (Fig. S5D). Upon exposure 428 to $\mathrm{CNO}$, however, the pupils of $\mathrm{Cre}^{+} / \mathrm{hM}^{+} \mathrm{D}^{+}$mice drastically constricted (Fig. S5D). These 429 data show that in floxed-Stop-hM3D mouse the robust activation of RGCs (as measured 430 by the dramatic change in pupil size) occurs only in the presence of both Cre activity and 431 CNO. Our data are in alignment with recent studies demonstrating that activation of $432 \mathrm{hM}$ 3q expressing RGCs (virally transduced) in vivo generates a near complete pupillary 433 constriction (Milosavljevic et al., 2016; Keenan et al., 2016; 2017; Seabrook et al., 2017).

\section{Selective activation of Cdh3-RGC increases pupil size}

436 Next, to selectively express hM3D in Cdh3-RGCs, we used the Cre-DOG (dependent on 437 GFP) system (Tang et al., 2015) to induce Cre recombinase activity specifically in Cdh3438 RGCs $\left(\mathrm{GFP}^{+}\right)$. Cre-DOG is a split Cre system that uses GFP as a scaffold to assemble and 439 induce Cre recombinase activity (schematized in Fig. 7A; Tang et al., 2015). In the retina, 440 AAVs encoding the GFP-dependent split-Cre components (N-Cre and C-Cre) can be used 441 to turn on Cre-dependent-reporter tdTomato specifically in GFP expressing RGCs 442 (schematized in Fig. 7B, 7C). We previously showed that the retrofitting of Cdh3-RGCs 443 using this system is highly selective (Tang et al., 2015). Indeed, we found that in the Cdh3444 GFP mouse line nearly all tdTomato expressing RGCs were also GFP+ [Fig. 7D-D”; 445 previously quantified: $\sim 85 \%$ of Tdtomato $^{+}$RGCs are also GFP $^{+}$(Tang et al., 2015)]. 446 TdTomato-expressing RGC axons in retrofitted Cdh3-GFP mice project to the same targets 447 as Cdh3-RGCs and completely overlap with the axonal projections of Cdh3-RGCs (Fig. 448 7E-F2"). Importantly, in the absence of GFP (GFP- retinas), no TdTomato expression was 449 observed (previously reported in Tang et al., 2015). Together, these data further validate 450 the high degree of specificity of the Cre-DOG system.

451 To test the role of Cdh3-RGCs in PLR behavior, we bred the Cdh3-GFP mouse line 452 to floxed-Stop-hM3D mouse line to create Cdh3-GFP ${ }^{+}$; floxed-Stop-hM3D mice. Next, we 453 injected a mixture of two AAVs that each individually encode N-Cre and C-Cre (the GFP- 
454 dependent split-Cre components) into the eyes of Cdh3-GFP ${ }^{+}$; floxed-Stop-hM3D mice 455 (schematized in Fig. 7G). After 5-6 weeks, the pupils of these mice were recorded prior to, 456 and after, CNO administration (pre- and post-CNO respectively; Fig. 7H). CNO 457 administration alone did not significantly change the pupil size of control animals [Cdh3$458 \mathrm{GFP}^{+}$; floxed-Stop-hM3D (AAV un-injected); p=0.99; n=9 mice; Fig. 7I, 7J] but CNO 459 mediated activation of hM3D expressing Cdh3-RGCs resulted in a significant increase in 460 pupil size [ 10\% increase in $\mathrm{Cdh} 3-\mathrm{GFP}^{+}$; $\mathrm{hM}^{+}$; $\mathrm{Cre}^{+}$mice; p<0.0001; Fig. 7I, 7J]. 461 Although the activation of Cdh3-RGCs resulted in a highly significant dilation of the pupils 462 the effect is rather small $(\sim 10 \%)$. We attribute this minor effect to the low efficiency of the 463 Cre-DOG technique with only a very small fraction of Cdh3-RGCs being converted into 464 Cre-expressing cells (Tang et al., 2015). Regardless, these results suggest that OPN core465 projecting Cdh3-RGCs play a unique role in controlling pupil size. Our data suggest a 466 putative "push-pull" system for controlling pupil aperture. The M1-ipRGCs projecting to 467 the OPN-shell drive pupil constriction (push) (Güler et al., 2008 and see above) whereas 468 the Cdh3-RGCs projecting to the OPN-core driving pupil dilation (pull) and thereby 469 dynamically control the full range of PLR behavior. These data, therefore, represent a step 470 towards dissecting the specific function of different types of ipRGCs and in doing so, 471 provide new insight into the distinct functional roles of the different OPN anatomical 472 compartments and pathways.

\section{Discussion}

475 Our study reveals a previously unrecognized topographically-non-uniform set of RGCs 476 controlling a discrete non-image-forming visual function: pupil size. We also discovered 477 the molecular underpinnings of this circuitry. That, in turn, unveiled mechanistic aspects 478 of their specification and a novel functional component of the visual circuit responsible for 479 pupil reflexes.

481 Tbx20 plays a role in establishing non-image-forming parallel optic pathways

482 Tbx20 is a highly conserved gene both in terms of its structure and function. Tbx20 is 483 known to play an essential role in cardiogenesis in both vertebrate and invertebrate species 484 (Pocock et al., 2008; Jensen et al., 2013; Greulich et al., 2011) and can function as both a 
485 transcriptional activator or a transcriptional repressor. Its activity is involved in multiple 486 aspects of cardiac cell proliferation and specification, and structural formation of the heart 487 and mutations in Tbx20 are associated with human congenital heart defects (Cai et al., 488 2005, 2013; Stennard et al., 2005; Takeuchi et al., 2005; Singh et al., 2005; Sakabe et al., 489 2012; Kirk et al., 2007; Liu et al., 2008; Posch et al., 2010). The role of Tbx20 in the 490 mammalian nervous system is barely understood, as very limited work has focused on it. 491 Tbx20 was shown by Pfaff and co-workers to be critical for normal development of the 492 hindbrain (Song et al., 2006) by controlling proper migration of facial neurons to their 493 correct destinations in the developing hindbrain, but not required for their initial 494 specification or patterning. Recently, Mason and colleagues identified Tbx20 as a marker 495 of contralateral-projecting RGCs during embryonic development (Wang et al., 2016). 496 Consistent with the findings in this study (Fig. 1 and Fig. S1), their data also shows that 497 Tbx20 is expressed in a subpopulation of RGCs and is graded along the dorsal-ventral axis.

Our results identify Tbx20 as being important for the assembly of non-image500 (see Fig. 2M-R in Seabrook et al., 2017) and Cdh3-RGCs are already born by this 501 developmental stage (Osterhout et al., 2014). Therefore, the loss of Tbx20-RGCs together 502 with the reduction in retinal innervation of non-imaging-forming brain targets in the Tbx20 503 conditional knockout mice supports the idea that Tbx20 promotes survival rather than 504 specification of different RGC types. Moreover, these differences in phenotypic traits, i.e. 505 loss of neurons in the visual system and migration deficits but not cell loss in the 506 somatosensory system, suggest that Tbx20 can play different roles within different sensory 507 systems. More broadly, this underscores the importance of studying the same genetic 508 program in different sensory systems, as this can reveal new facets and rules governing 509 nervous system development and function.

510 Previous studies identified the transcription factor T-box family protein Tbr2 (also 511 called 'Eomes') and the POU-domain protein Brn3b as necessary for establishing the 512 central projection pathways of non-image-forming RGCs, including all ipRGCs (Quina et 513 al.2005; Badea et al., 2009; Sweeney et al., 2014; Mao et al., 2014; Seabrook et al., 2017). 514 Tbx20 most likely functions downstream of both Tbr2 and Brn3b because i) majority of 515 Tbx20-RGCs express both these factors and ii) Tbx20-RGCs represent a subset of all 
516 ipRGCs. Indeed, a recent study by Badea and colleagues (Sajgo et al., 2017) also found

517 that normally Tbx20 is highly enriched in RGCs that express Brn3b but its expression is

518 nearly absent when Brn3b function is lost. In the future, we plan to explore the genes

519 regulated by Tbx20 transcriptional activity as well as the consequences of deleting Tbx20

520 from the adult visual system.

521

522 Tbx20-RGCs are minor contributors to the constriction phase of the PLR

523 M1 ipRGCs and their connections to neurons in the OPN shell are critical for driving the 524 pupillary reflex (Güler et al., 2008). Previous studies demonstrated that shell neurons are

525 highly activated in response to light stimuli and that the Edinger Westfal nucleus- which is

526 the downstream target structure of the OPN, receives inputs predominantly from the OPN

527 neurons whose somas reside in the shell (Prichard et al., 2002; Baver et al., 2008). Although

528 ablating all shell-projecting M1 ipRGCs leads to a severe attenuation of the PLR ( 42\%

529 reduction in pupil constriction at high light levels, and no constriction at low light levels),

530 light-induced pupil constriction is not completely abolished in the absence of M1s (Güler

531 et al., 2008). Complete loss of the PLR only occurs when all ipRGCs, as well as rod/cone

532 mediated signaling, are eliminated (Hattar et al., 2003; Panda et al., 2003; Badea et al.,

533 2009; Hatori et al., 2008, Sweeney et al., 2014; Mao et al., 2014). Removing M1s and all

$534 \mathrm{rod} /$ cone signaling is effectively the same as removing all RGCs. Thus, RGCs other than

535 M1 ipRGCs projecting to the OPN shell must contribute to the PLR.

536 Conditionally knocking out Tbx20 eliminated a subset of RGCs that project to the

537 OPN core as well as the OPN shell. Alone this manipulation did not lead to a severe deficit

538 in light-evoked pupillary constriction. This is not surprising, as the population of M1

539 ipRGCs that do not express Tbx20 remain intact in Tbx20 cKO mice. Indeed, even an

540 extremely small number of retinal inputs to the OPN ( 7-20\%) is sufficient to drive a strong

541 PLR response (Güler et al., 2008; Mao et al., 2014; Seabrook et al., 2017). A key

542 contribution of Tbx20-RGCs to the pupillary reflex was revealed, however, when

543 melanopsin-mediated signaling was abolished in Tbx20 cKO mice. Depending on the

544 intensity of the light stimulus, pupillary constriction in those mice was reduced $\sim 45-55 \%$

545 in Tbx20/Mel dKO mice as compared to control mice. The level of pupillary constriction

546 deficits observed in Tbx20/Mel dKO mice are comparable to those observed when all shell- 
547 projecting M1 ipRGCs are ablated (Güler et al., 2008). Our experimental data cannot 548 precisely disambiguate the specific role of OPN-core versus OPN-shell in the PLR, as 549 retinal innervation to both these targets is compromised in Tbx $20 \mathrm{cKO}$ mice. However, the 550 most parsimonious explanation for the dramatic disruption of the PLR in Tbx20/Mel 551 double $\mathrm{KO}$ is that it is primarily due to the loss of M1 ipRGC (Tbx $\left.20^{+}\right)$innervation to the 552 OPN-shel,l compounded by the loss of melanopsin signaling in the remaining OPN-shell 553 projecting M1 ipRGCs (Tbx20-).

\section{Conventional-photoreceptor driven visual signals contribute to the PIPR}

556 Our analysis of the pupillary reflex in Tbx20/Mel dKO mice unveiled a novel role of $557 \mathrm{rod} /$ cone mediated signaling in generating the post-illumination pupillary reflex (PIPR)558 which is the persistent constriction of the pupil after the offset of a relatively high-intensity 559 light stimulus. The PIPR is widely used as an indicator of ipRGC health in humans and 560 non-human primates as a diagnostic for a broad range of diseases including glaucoma, 561 multiple sclerosis, and seasonal affective disorder (Meltzer et al., 2017, Feigl \& Zele 2014).

562 The PIPR is thought to be driven by the sustained activity of ipRGCs after the offset of 563 light. Indeed, a recent report showed that in the presence of a truncated version of 564 melanopsin lacking residues beyond amino acid 397, mice fail to maintain pupillary 565 constriction after light offset (Mure et al., 2016). The circuit-based mechanistic 566 underpinnings of the PIPR has not been examined. We found that similar to primates, the 567 PIPR of mice is strongly dependent on melanopsin signaling, as Mel KO failed to maintain 568 a sustained pupillary constriction after the offset of a relatively high irradiance stimulus. 569 However, rodent ipRGCs show persistent activity, albeit attenuated, even in the absence of 570 the melanopsin signaling (Schmidt et al., 2014; Wong 2012). Indeed, we found that the 571 pupillary escape from constriction after light offset was significantly greater in the 572 Tbx20/Mel dKO mice in comparison to mice lacking only melanopsin signaling. This 573 suggests that OPN-projecting ipRGCs use both melanopsin-mediated as well as rod/cone574 mediated visual signals to generate the PIPR.

\section{Insights into the role of the OPN 'core' pathway}

577 The OPN receives inputs from multiple types of RGCs namely, M1 and M2 ipRGCs and 
578 from 'diving' RGCs. Each of these RGC types is capable of encoding and relaying 579 irradiance changes necessary for driving the PLR. However, our data and the work of 580 others indicate that M1 ipRGC inputs to the shell provide the main drive for pupillary 581 constriction and are sufficient to drive near normal PLR. This raises the question of the 582 purpose of the retina-OPN-core pathway. We selectively probed the functional output of 583 the OPN-core projecting Cdh3-RGCs by reversibly manipulating their activity using 584 chemogenetic tools and found that Cdh3-RGC activation, in fact, results in a modest 585 dilation of the pupil. This suggests that the OPN-shell and OPN-core function in a "push586 pull" manner to modulate the aperture of the eye. Indeed, OPN inputs and outputs of 587 extremely heterogeneous across multiple species (Gamlin 2006). The M1 ipRGC-to588 OPNshell-to-EW pathway is part of the parasympathetic system and has received a lot of attention due to its role in generating light-induced pupil constriction. However, OPN 590 neurons also project to several other nuclei including the SC, periaqueductal gray, nucleus

591 and nucleus of the posterior commissure, brain targets have previously been implicated as 592 part of the sympathetic pathway involved in controlling pupil dilation and arousal states 593 (Klooster et al., 1995; Wang and Munoz, 2015; Salay et al., 2018). In future work, it will 594 be important to dissect the downstream targets and other inputs to OPN-core neurons as a 595 way to parse the contributions of this circuit to pupil size dynamics in the context of both 596 light-mediated and light-independent arousal.

\section{Methods}

Animals

601 Experimental procedures were in accordance with NIH guidelines and approved by the 602 Institutional Animal Care and Use Committees at UCSD and Stanford University School 603 of Medicine. The generation and characterization of Tbx20 floxed and Tbx20-GFP BAC 604 transgenic mouse lines were described previously (Cai et al., 2005 and Shen et al., 2011, 605 respectively). Tryptophan hydroxylase 2-Cre (Tph2-Cre) mice were obtained from 606 MMRRC (https://www.mmrrc.org/catalog/sds.php?mmrrc_id=36634) (Osterhout et al., 607 2011; 2014). Cadherin-3-EGFP (Cdh3-GFP) were obtained from MMRRC and previously 608 characterized (Osterhout et al., 2011; 2014). Homeobox d10 BAC-EGFP (Hoxd10-GFP) 
609 mice were obtained from MMRRC and described previously in Dhande et al., 2013. Opn4 610 (opsin 4/melanopsin) Tau-LacZ mice were obtained from Jackson Laboratory 611 (https://www.jax.org/strain/021153) and reported previously (Hattar et al., 2002; 2006). 612 Opn4 Tau-LacZ homozygous mice do not express melanopsin (Mel KO). CAG-LSL$613 \mathrm{hM} 3 \mathrm{Dq} / \mathrm{mCitrine}$ transgenic mice were obtained from the Jackson Laboratory 614 (https://www.jax.org/strain/026220) and reported previously (Zhu et al., 2106). These mice 615 express the hM3Dq DREADD and mCitrine reporter after Cre-recombinase mediated 616 excision of a floxed-Stop cassette.

\section{Histology \& Immunohistochemistry}

619 Mice were transcardially perfused, brains harvested and postfixed overnight $(\sim 12 \mathrm{hrs})$ in $6204 \%$ PFA at $4{ }^{\circ} \mathrm{C}$, immersed in $30 \%$ sucrose for $2-3$ days at $4{ }^{\circ} \mathrm{C}$, and sectioned at $45 \mu \mathrm{m}$ on 621 a sliding microtome. Tissue was incubated overnight at $4^{\circ} \mathrm{C}$ in primary antibodies and 622 incubated for $2 \mathrm{hrs}$ at room temperature with appropriate secondary antibodies. Retinas 623 were harvested and processed for immunohistochemistry as previously described 624 (Huberman et al., 2008; Tang et al., 2015).

625 Primary antibodies used were: rabbit anti-GFP (1:1,000, Molecular Probes Cat\# A626 6455, RRID:AB_221570), chicken anti-GFP (1:1,000; Aves Labs Cat\# GFP-1020, 627 RRID:AB_10000240), rabbit anti-Cart (1:1,000, Phoenix Pharmaceuticals Cat\# H-003-62, 628 RRID:AB_2313614), mouse SMI-32 (1:1,000, Covance Research Products Inc Cat\# SMI629 32P, RRID:AB_2314912), rabbit or guinea-pig anti-Tbx20 (1:1,000; Song et al., 2006), 630 rabbit anti-melanopsin (1:1,000; Advanced Targeting Systems Cat\# AB-N39, 631 RRID:AB_1608076), rabbit anti-Tbr2 (1:1,000, Abcam Cat\# ab23345, 632 RRID:AB_778267), mouse anti- $\beta$-Galactosidase ( $\beta$-Gal) $(1: 1,000$, Sigma-Aldrich Cat\# 633 G8021, RRID:AB_259970), rabbit anti-dsRed (1:1,000, Clontech Laboratories, Inc. Cat\# 634 632496, RRID:AB_10013483), rabbit anti-Brn3b (1:1,000, Quina et al., 2005). Species635 specific secondary antibodies conjugated to Alexa Fluor 488, 594 or 647 (1: 1,000; 636 Invitrogen and Jackson Laboratory) were used.

\section{$638 \quad X$-gal staining}

639 Tissue was harvested and sectioned as described above, with the exception that brains were 
640 fixed for $4 \mathrm{hrs}$ in 4\%PFA. X-gal staining was performed as previously described (Dhande 641 et al., 2012). Control and Tbx20 cKO mice that were also heterozygous for the Opn4 Tau642 LacZ allele were used for X-gal staining.

644 RGC marker and density quantification

645 Quantification of $\mathrm{GFP}^{+}$RGCs and expression of molecular markers was performed as 646 previously described (Dhande et al., 2013). For marker analysis, labeled RGCs were 647 counted in both the central and peripheral region of a randomly selected retinal leaflet. 648 Counts were performed by manual cell identification using Neurolucida 649 (MicroBrightField).

651 In situ hybridization and quantification

652 Mice were transcardially perfused, retinas harvested and postfixed overnight in 4\% PFA at $6534^{\circ} \mathrm{C}$, immersed in $30 \%$ sucrose for one day at $4^{\circ} \mathrm{C}$, embedded in Tissue-Tek O.C.T. 654 compound (Sakura) and sectioned at $15 \mu \mathrm{m}$ on a cryostat. RNAscope Multiplex Fluorescent 655 Assay was performed according to manufacturer's instructions using custom Tbx20 probes 656 (targeting the 460-1708 region of NM_194263.2; Cat No. 511991; Advanced Cell

657 Diagnostics). Images were acquired with a Zeiss LSM 880 or a Zeiss AxioScan 658 microscope. Fluorescently labeled cells were manually counted from 5 consecutive 659 sections per animal.

660 Intravitreal injections of tracers or viruses

661 Binocular injections of $1-2 \mu \mathrm{L}$ of Cholera Toxin- $\beta$ conjugated to Alexa Fluor488 (CT $\beta$ 662488 , Invitrogen) or AAV-iCre (Vector BioLabs) or a 1:1 mix of AAV2/1.EF1a.N-

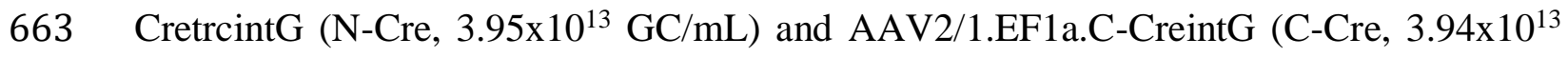
664 GC/mL, Gene Transfer Vector Core, Harvard Medical School). CT $\beta-488$ injected mice 665 were killed two days later, and virus injected mice were killed after behavioral analyses. 666 Brain tissue and retinas were harvested and processed as described above (see Histology \& 667 immunohistochemistry).

670 Flat mount retinas with labeled Tbx20-GFP or Cdh3-GFP cells were outlined in Image J 
671 to demarcate tear locations. Cells were identified based on the signal intensity and cell

672 body positions were recorded using the ImageJ ROI manager. These parameters were

673 imported into the Retistruct package in R (Sterratt et al., 2013; R Development Core Team,

674 2014). Flat mount retinas were then reconstructed to standard spherical retina space and

675 labeled cells were plotted in spherical coordinates. Isodensity contour lines were derived

676 using kernel density estimates. Cell counts were obtained from each retinal pole. For

677 comparing cell counts in opposing retinal poles, binomial tests with the stats $\mathrm{R}$ package

678 were performed for each retina. To compare average cell counts in opposing poles, paired

679 t-tests were performed.

680

681 Quantification of target area and innervation density

682 Measurements were taken from 3-5 tissue sections depending on the target size. CT $\beta-488$

683 or GFP labeling was used to outline the boundaries of the retinorecipient targets. A 684 template boundary based on representative control OPN and SCN boundaries was used for 685 measuring $\beta$-Gal staining density. ImageJ 'Area' and 'Integrated density' tools were used 686 to measure the retinorecipient target area and density of innervation (product of mean gray 687 pixel value and target area).

688

689 Pupillometry

690 The consensual PLR of dark-adapted mice was recorded and quantified as follows. After

691 2hrs of dark-adaption, under infrared lighting conditions, the left eye of the awake hand-

692 restrained mouse was illuminated with a light intensity of 100, 1,000 or 10,000 lux using a

693 single $470 \mathrm{~nm}$ tunable LED (Thor Labs, M470L3) for 30s. The consensual (right eye) was

694 recorded using a Sony handycam immediately before illumination, during illumination

695 (30s), and for 1 minute after the LED turned off. 3-4 recordings for each lighting condition

696 were made from each mouse between Zeitgeber time (ZT)6 and ZT10 (where ZT0 is lights-

697 on and ZT12 is lights-off). To induce complete ciliary constriction, $100 \mathrm{mM}$ Carbachol

698 was prepared and applied as previously described (Lucas et al., 2003).

699 For quantification of pupil size, individual frames were extracted from the video 700 recordings at every $5 \mathrm{~s}$ from $\mathrm{t}=-5 \mathrm{sec}$ (dark adapted, lights off), to $\mathrm{t}=90 \mathrm{sec}$. Pupil diameter

701 was calculated for each of these frames and normalized to the diameter of the eye in the 
702 same frame in ImageJ. Pupil area was calculated for each time point relative to the initial

703 dark-adapted pupil size at $\mathrm{t}=-5 \mathrm{sec}$. Chemogenetic induced changes in pupil size were

704 recorded and quantified as described above, with the following modifications: under

705 constant dark conditions (no LED stimulus) images of the right pupil were captured before

706 and 40 minutes after intraperitoneal administration of Clozapine $\mathrm{N}$-oxide (CNO, 5mg/kg,

707 Tocris Bioscience). Post-CNO pupil size was normalized to pre-CNO pupil size.

709 Targeting Cdh3-RGCs using the Cre-DOG system

710 The Cre-DOG system was used to selectively infect Cdh3-GFP RGCs with Cre as in: Tang 711 et al. (2015). Briefly, intraocular injections of three AAVs each encoding either N-Cre, C-

712 Cre or flex-TdTomato-reporter, were made into 2-3 month old Cdh3-GFP mice. 2-4 weeks

713 later, retinas and brains were harvested and processed as described above (see Histology \& 714 immunohistochemistry). For chemogenetic experiments, $1-2 \mu \mathrm{L}$ of a 1:1 combination of 715 AAV N-Cre and AAV C-Cre viruses were injected binocularly into mice carrying both the 716 CAG-floxed-Stop-hM3D/mCitrine transgene and the Cdh3-GFP transgene. Two rounds of 717 retinal injections were performed one week apart. Pupillometry was performed 5-6 weeks 718 after the last set of retinal injections animals.

\section{Experimental design and statistical analysis}

721 For behavioral analyses, 2-3 month old mice were used. For all other experiments, mice 722 aged postnatal day (P)19 - P28 were used, unless otherwise stated. All mice were 723 maintained on a mixed background. Mice of both sexes were used in all experiments. All 724 data are expressed as Mean \pm Standard Error of Mean (SEM). Data were significant when $725 \mathrm{p}<0.05$, as determined by a two-way ANOVA with Bonferroni post-hoc test for PLR 726 analysis, and by Student's t-test for all other analyses, using GraphPad (Prism program, 727 California, USA). Paired tests were used to compare within-group repeated measure data. 728 


\section{Author Contributions}

734 O.S.D. and A.D.H conceived and designed the experiments and wrote the paper. O.S.D.

735 and T.A.S. characterized the Tph2Cre mouse line. O.S.D., A.H.P., N.I., and P.L.N.

736 performed brain histology. O.S.D., A.H.P. and N.I. performed behavior experiments.

737 O.S.D and A.H.P. imaged and analyzed histology and behavior data. L.D.S. analyzed

738 retinal gradients. J.T.W. performed initial molecular screen. S.E. provided the Tbx20

739 conditional allele mice and Tbx20-GFP mice. O.S.D. performed retinal histology;

740 performed and analyzed chemogenetic experiments; and prepared the figures and

741 manuscript.

742

\section{Acknowledgements}

744 This work was supported by a Knights Templar Eye Foundation Grant (O.S.D.), NIH RO1

745 EY022157 (A.D.H.), NIH RO1 EY026100 (A.D.H.), and a Pew Scholar Award (A.D.H.).

746 We thank Dr. Samuel Pfaff and Dr. Eric Turner for kindly providing Tbx20 and Brn3b

747 antibodies and Dr. Rana El-Danaf and Dr. Supraja Varadarajan for helpful comments on

748 an earlier draft on the manuscript.

749

\section{Declaration of Interests}

751 The authors declare no competing interests.

752

\section{References}

754 Baver SB, Pickard GE, Sollars PJ, Pickard GE (2008) Two types of melanopsin retinal

755 ganglion cell differentially innervate the hypothalamic suprachiasmatic nucleus and the

756 olivary pretectal nucleus. Eur J Neurosci 27:1763-1770.

757

758

759

760

761

762

763
Berson DM, Dunn FA, Takao M (2002) Phototransduction by retinal ganglion cells that set the circadian clock. Science 295:1070-1073.

Berson DM, Castrucci AM, Provencio I (2010) Morphology and mosaics of melanopsinexpressing retinal ganglion cell types in mice. J Comp Neurol 518:NA-NA.

Cai C-L, Zhou W, Yang L, Bu L, Qyang Y, Zhang X, Li X, Rosenfeld MG, Chen J, Evans S (2005) T-box genes coordinate regional rates of proliferation and regional specification during cardiogenesis. Development 132:2475-2487. 
764 Cai X, Zhang W, Hu J, Zhang L, Sultana N, Wu B, Cai W, Zhou B, Cai C-L (2013)

765 Tbx20 acts upstream of Wnt signaling to regulate endocardial cushion formation and

766 valve remodeling during mouse cardiogenesis. Development 140:3176-3187.

767 Chen S-K, Badea TC, Hattar S (2011) Photoentrainment and pupillary light reflex are

768 mediated by distinct populations of ipRGCs. Nature 476:92-95.

769 Crespo D, O'Leary DDM, Maxwell Cowan W (1985) Changes in the numbers of optic

770 nerve fibers during late prenatal and postnatal development in the albino rat.

771 Dhande OS, Estevez ME, Quattrochi LE, El-Danaf RN, Nguyen PL, Berson DM,

772

773 Huberman AD (2013) Genetic dissection of retinal inputs to brainstem nuclei controlling image stabilization. J Neurosci 33:17797-17813.

774

775

776

777

778

779

780

781

782

783

784

785

786

787

788

789

790

791

792

793

794

795

796

Dhande OS, Huberman AD (2014) Retinal ganglion cell maps in the brain: implications for visual processing. Curr Opin Neurobiol 24:133-142.

Dhande OS, Bhatt S, Anishchenko A, Elstrott J, Iwasato T, Swindell EC, Xu H-P, Jamrich M, Itohara S, Feller MB, Crair MC (2012) Role of adenylate cyclase 1 in retinofugal map development. J Comp Neurol 520:1562-1583.

Dhande OS, Stafford BK, Lim J-HA, Huberman AD (2015) Contributions of retinal ganglion cells to subcortical visual processing and behaviors. Annu Rev Vis Sci $1: 291-328$.

Distler C, Hoffmann KP (1989) The pupillary light reflex in normal and innate microstrabismic cats, I: Behavior and receptive-field analysis in the nucleus praetectalis olivaris. Vis Neurosci 3:127-138.

Distler C, Hoffmann KP (1989) The pupillary light reflex in normal and innate microstrabismic cats, II: Retinal and cortical input to the nucleus praetectalis olivaris. Vis Neurosci 3:139-153.

El-Danaf RN, Huberman AD (2018) Sub-topographic maps for regionally enhanced analysis of visual space in the mouse retina. J Comp Neurol.

Feigl B, Zele AJ (2014) Melanopsin-expressing intrinsically photosensitive retinal ganglion cells in retinal disease. Optom Vis Sci 91:894-903.

Fox MA, Guido W (2011) Shedding Light on Class-Specific Wiring: Development of Intrinsically Photosensitive Retinal Ganglion Cell Circuitry. Mol Neurobiol 44:321329.

Gamlin PDR (2006) The pretectum: connections and oculomotor-related roles. Prog Brain Res 151:379-405. 
797

798

799

800

801

802

803

804

805

806

807

808

809

810

811

812

813

814

815

816

817

818

819

820

821

822

823

824

825

826

827

828

829

830

831

832

Gamlin PDR, Clarke RJ (1995) The pupillary light reflex pathway of the primate. J Am Optom Assoc 66:415-418.

Gamlin PDR, McDougal DH, Pokorny J, Smith VC, Yau K-W, Dacey DM (2007) Human and macaque pupil responses driven by melanopsin-containing retinal ganglion cells. Vision Res 47:946-954.

Gamlin PD, Zhang H, Clarke RJ (1995) Luminance neurons in the pretectal olivary nucleus mediate the pupillary light reflex in the rhesus monkey. Exp Brain Res 106:169-176.

Gong S, Zheng C, Doughty ML, Losos K, Didkovsky N, Schambra UB, Nowak NJ, Joyner A, Leblanc G, Hatten ME, Heintz N (2003) A gene expression atlas of the central nervous system based on bacterial artificial chromosomes. Nature 425:917925.

Greulich F, Rudat C, Kispert A (2011) Mechanisms of T-box gene function in the developing heart. Cardiovasc Res 91:212-222.

Güler AD, Ecker JL, Lall GS, Haq S, Altimus CM, Liao H-W, Barnard AR, Cahill H, Badea TC, Zhao H, Hankins MW, Berson DM, Lucas RJ, Yau K-W, Hattar S (2008) Melanopsin cells are the principal conduits for rod-cone input to non-image-forming vision. Nature 453:102-105.

Hatori M, Le H, Vollmers C, Keding SR, Tanaka N, Schmedt C, Jegla T, Panda S (2008) Inducible Ablation of Melanopsin-Expressing Retinal Ganglion Cells Reveals Their Central Role in Non-Image Forming Visual Responses Hendricks M, ed. PLoS One 3:e2451.

Hattar S, Liao HW, Takao M, Berson DM, Yau KW (2002) Melanopsin-containing retinal ganglion cells: architecture, projections, and intrinsic photosensitivity. Science 295:1065-1070.

Hattar S, Lucas RJ, Mrosovsky N, Thompson S, Douglas RH, Hankins MW, Lem J, Biel M, Hofmann F, Foster RG, Yau KW (2003) Melanopsin and rod—cone photoreceptive systems account for all major accessory visual functions in mice. Nature 424:76-81.

Hattar S, Kumar M, Park A, Tong P, Tung J, Yau K-W, Berson DM (2006) Central projections of melanopsin-expressing retinal ganglion cells in the mouse. J Comp Neurol 497:326-349.

Huberman AD, Manu M, Koch SM, Susman MW, Lutz AB, Ullian EM, Baccus SA, Barres BA (2008) Architecture and activity-mediated refinement of axonal projections from a mosaic of genetically identified retinal ganglion cells. Neuron 59:425-438. 
833 Huberman AD, Wei W, Elstrott J, Stafford BK, Feller MB, Barres BA (2009) Genetic

$834 \quad$ Identification of an On-Off Direction- Selective Retinal Ganglion Cell Subtype

835 Reveals a Layer-Specific Subcortical Map of Posterior Motion. Neuron 62:327-334.

836

837

838

839

840

841

842

843

844

845

846

847

848

849

850

851

852

853

854

855

856

857

858

859

860

861

862

863

864

865

866

867

Jensen B, Wang T, Christoffels VM, Moorman AFM (2013) Evolution and development of the building plan of the vertebrate heart. Biochim Biophys Acta - Mol Cell Res 1833:783-794.

Kay JN, De la Huerta I, Kim I-J, Zhang Y, Yamagata M, Chu MW, Meister M, Sanes JR (2011) Retinal ganglion cells with distinct directional preferences differ in molecular identity, structure, and central projections. J Neurosci 31:7753-7762.

Keenan WT, Fernandez DC, Shumway LJ, Zhao H, Hattar S (2017) Eye-drops for activation of DREADDs. Front Neural Circuits 11.

Keenan WT, Rupp AC, Ross RA, Somasundaram P, Hiriyanna S, Wu Z, Badea TC, Robinson PR, Lowell BB, Hattar SS (2016) A visual circuit uses complementary mechanisms to support transient and sustained pupil constriction. Elife 5.

Kim H-L, Jeon JH, Koo T-H, Lee U-Y, Jeong E, Chun M-H, Moon J-I, Massey SC, Kim I-B (2012) Axonal synapses utilize multiple synaptic ribbons in the mammalian retina. Fox MA, ed. PLoS One 7:e52295.

Kim I-J, Zhang Y, Meister M, Sanes JR (2010) Laminar Restriction of Retinal Ganglion Cell Dendrites and Axons: Subtype-Specific Developmental Patterns Revealed with Transgenic Markers. J Neurosci 30:1452-1462.

Kirk EP et al. (2007) Mutations in cardiac T-box factor gene TBX20 are associated with diverse cardiac pathologies, including defects of septation and valvulogenesis and cardiomyopathy. Am J Hum Genet 81:280-291.

Klooster J, Vrensen GFJM, Müller LJ, van der Want JJL (1995) Efferent projections of the olivary pretectal nucleus in the albino rat subserving the pupillary light reflex and related reflexes a light microscopic tracing study. Brain Res 688:34-46.

Lim E-J, Kim I-B, Oh S-J, Chun M-H (2007) Identification and characterization of SMI32-immunoreactive amacrine cells in the mouse retina. Neurosci Lett 424:199202.

Lim JHA, Stafford BK, Nguyen PL, Lien B V., Wang C, Zukor K, He Z, Huberman AD (2016) Neural activity promotes long-distance, target-specific regeneration of adult retinal axons. Nat Neurosci 19:1073-1084.

Liu C, Shen A, Li X, Jiao W, Zhang X, Li Z (2008) T-box transcription factor TBX20 mutations in Chinese patients with congenital heart disease. Eur J Med Genet 51:580-587. 
868 Lucas RJ, Douglas RH, Foster RG (2001) Characterization of an ocular photopigment

869 capable of driving pupillary constriction in mice. Nat Neurosci 4:621-626.

870 Lucas RJ, Hattar S, Takao M, Berson DM, Foster RG, Yau K-W (2003) Diminished

871 pupillary light reflex at high irradiances in melanopsin-knockout mice. Science

$872 \quad 299: 245-247$.

873 Maayani S, Treister G, Sokolovsky M (1975) Drugs affecting the cholinergic system in

874 the intact mammalian eye. I. Evaluation of the miotic activity of acetylcholine-like

875 drugs in the mouse eye. Invest Ophthalmol 14:232-235.

876

877

878

879

880

881

882

883

884

885

886

887

888

889

890

891

892

893

894

895

896

897

898

899

900

901

902

Mao C-A, Li H, Zhang Z, Kiyama T, Panda S, Hattar S, Ribelayga CP, Mills SL, Wang SW (2014) T-box transcription regulator Tbr2 is essential for the formation and maintenance of Opn4/melanopsin-expressing intrinsically photosensitive retinal ganglion cells. J Neurosci 34:13083-13095.

McNeill DS, Sheely CJ, Ecker JL, Badea TC, Morhardt D, Guido W, Hattar S (2011) Development of melanopsin-based irradiance detecting circuitry. Neural Dev 6:8.

Meltzer E, Sguigna P V., Subei A, Beh S, Kildebeck E, Conger D, Conger A, Lucero M, Frohman BS, Frohman AN, Saidha S, Galetta S, Calabresi PA, Rennaker R, Frohman TC, Kardon RH, Balcer LJ, Frohman EM (2017) Retinal Architecture and Melanopsin-Mediated Pupillary Response Characteristics. JAMA Neurol 74:574.

Milosavljevic N, Cehajic-Kapetanovic J, Procyk CA, Lucas RJ (2016) Chemogenetic Activation of Melanopsin Retinal Ganglion Cells Induces Signatures of Arousal and/or Anxiety in Mice. Curr Biol 26:2358-2363.

Mure LS et al. (2009) Melanopsin Bistability: A fly's eye technology in the human retina Egles C, ed. PLoS One 4:e5991.

Mure LS, Hatori M, Zhu Q, Demas J, Kim IM, Nayak SK, Panda S (2016) Melanopsinencoded response properties of intrinsically photosensitive retinal ganglion cells. Neuron 90:1016-1027.

Osterhout JA, El-Danaf RN, Nguyen PL, Huberman AD (2014) Birthdate and outgrowth timing predict cellular mechanisms of axon target matching in the developing visual pathway. Cell Rep 8:1006-1017.

Osterhout JA, Josten N, Yamada J, Pan F, Wu S, Nguyen PL, Panagiotakos G, Inoue YU, Egusa SF, Volgyi B, Inoue T, Bloomfield SA, Barres BA, Berson DM, Feldheim DA, Huberman AD (2011) Cadherin-6 mediates axon-target matching in a nonimage-forming visual circuit. Neuron 71:632-639.

Panda S, Provencio I, Tu DC, Pires SS, Rollag MD, Castrucci AM, Pletcher MT, Sato TK, Wiltshire T, Andahazy M, Kay SA, Van Gelder RN, Hogenesch JB (2003) 
903

904

905

906

907

908

909

910

911

912

913

914

915

916

917

918

919

920

921

922

923

924

925

926

927

928

929

930

931

932

933

934

935

Melanopsin is required for non-image-forming photic responses in blind mice.

Science 301:525-527.

Pocock R, Mione M, Hussain S, Maxwell S, Pontecorvi M, Aslam S, Gerrelli D, Sowden JC, Woollard A (2008) Neuronal function of Tbx20 conserved from nematodes to vertebrates. Dev Biol 317:671-685.

Posch MG, Gramlich M, Sunde M, Schmitt KR, Lee SHY, Richter S, Kersten A, Perrot A, Panek AN, Al Khatib IH, Nemer G, Megarbane A, Dietz R, Stiller B, Berger F, Harvey RP, Ozcelik C (2010) A gain-of-function TBX20 mutation causes congenital atrial septal defects, patent foramen ovale and cardiac valve defects. J Med Genet 47:230-235.

Prichard JR, Stoffel RT, Quimby DL, Obermeyer WH, Benca RM, Behan M (2002) Fos immunoreactivity in rat subcortical visual shell in response to illuminance changes. Neuroscience 114:781-793.

R Development Core Team (2014) R: A Language and Environment for Statistical Computing. R Foundation for Statistical Computing, Vienna, Austria. R Foundation for Statistical Computing, Vienna

Sakabe NJ, Aneas I, Shen T, Shokri L, Park S-Y, Bulyk ML, Evans SM, Nobrega MA (2012) Dual transcriptional activator and repressor roles of TBX20 regulate adult cardiac structure and function. Hum Mol Genet 21:2194-2204.

Salay, LD, Ishiko, N, Huberman, AD (2018) A midline thalamic circuit determines reactions to visual threat. Nature.

Sand A, Schmidt TM, Kofuji P (2012) Diverse types of ganglion cell photoreceptors in the mammalian retina. Prog Retin Eye Res 31:287-302.

Sanes JR, Masland RH (2015) The Types of Retinal Ganglion Cells: Current Status and Implications for Neuronal Classification. Annu Rev Neurosci 38:221-246.

Schmidt TM, Alam NM, Chen S, Kofuji P, Li W, Prusky GT, Hattar S (2014) A role for melanopsin in alpha retinal ganglion cells and contrast detection. Neuron 82:781788.

Schmidt TM, Chen S-K, Hattar S (2011) Intrinsically photosensitive retinal ganglion cells: many subtypes, diverse functions. Trends Neurosci 34:572-580.

Seabrook TA, Dhande OS, Ishiko N, Wooley VP, Nguyen PL, Huberman AD (2017b) Strict Independence of Parallel and Poly-synaptic Axon-Target Matching during Visual Reflex Circuit Assembly. Cell Rep 21:3049-3064. 
936

937

938

939

940

941

942

943

944

945

946

947

948

949

950

951

952

953

954

955

956

957

958

959

960

961

962

963

964

965

966

967

968

969

970

971

Shen T, Aneas I, Sakabe N, Dirschinger RJ, Wang G, Smemo S, Westlund JM, Cheng H, Dalton N, Gu Y, Boogerd CJ, Cai C, Peterson K, Chen J, Nobrega MA, Evans SM (2011) Tbx20 regulates a genetic program essential to adult mouse cardiomyocyte function. J Clin Invest 121:4640-4654.

Singh MK, Christoffels VM, Dias JM, Trowe M-O, Petry M, Schuster-Gossler K, Bürger A, Ericson J, Kispert A (2005) Tbx20 is essential for cardiac chamber differentiation and repression of Tbx2. Development 132:2697-2707.

Song M-R, Shirasaki R, Cai C-L, Ruiz EC, Evans SM, Lee S-K, Pfaff SL (2006) T-Box transcription factor $\mathrm{Tbx} 20$ regulates a genetic program for cranial motor neuron cell body migration. Development 133:4945-4955.

Sterratt et al (2013) Standard Anatomical and Visual Space for the Mouse Retina: Computational Reconstruction and Transformation of Flattened Retinae with the Retistruct Package. PLoS Comput Biol. 9, (2): e1002921

Stennard FA, Costa MW, Lai D, Biben C, Furtado MB, Solloway MJ, McCulley DJ, Leimena C, Preis JI, Dunwoodie SL, Elliott DE, Prall OWJ, Black BL, Fatkin D, Harvey RP (2005) Murine T-box transcription factor Tbx20 acts as a repressor during heart development, and is essential for adult heart integrity, function and adaptation. Development 132:2451-2462.

Sweeney NT, Tierney H, Feldheim DA (2014) Tbr2 is required to generate a neural circuit mediating the pupillary light reflex. J Neurosci 34:5447-5453.

Sweeney NT, James KN, Nistorica A, Lorig-Roach RM, Feldheim DA (2017) Expression of transcription factors divides retinal ganglion cells into distinct classes. J Comp Neurol.

Takeuchi JK, Mileikovskaia M, Koshiba-Takeuchi K, Heidt AB, Mori AD, Arruda EP, Gertsenstein M, Georges R, Davidson L, Mo R, Hui C-C, Henkelman RM, Nemer M, Black BL, Nagy A, Bruneau BG (2005) Tbx20 dose-dependently regulates transcription factor networks required for mouse heart and motoneuron development. Development 132:2463-2474.

Tang JCY, Rudolph S, Dhande OS, Abraira VE, Choi S, Lapan SW, Drew IR, Drokhlyansky E, Huberman AD, Regehr WG, Cepko CL (2015) Cell type-specific manipulation with GFP-dependent Cre recombinase. Nat Neurosci 18:1334-1341.

Trejo LJ, Cicerone CM (1984) Cells in the pretectal olivary nucleus are in the pathway for the direct light reflex of the pupil in the rat. Brain Res 300:49-62.

Urban DJ, Roth BL (2015) DREADDs (Designer Receptors Exclusively Activated by Designer Drugs): Chemogenetic Tools with Therapeutic Utility. Annu Rev Pharmacol Toxicol 55:399-417. 
972 Viney TJ, Balint K, Hillier D, Siegert S, Boldogkoi Z, Enquist LW, Meister M, Cepko

973 CL, Roska B (2007) Local Retinal Circuits of Melanopsin-Containing Ganglion

$974 \quad$ Cells Identified by Transsynaptic Viral Tracing. Curr Biol 17:981-988.

975 Wang CA, Munoz DP (2015) A circuit for pupil orienting responses: Implications for cognitive modulation of pupil size. Curr Opin Neurobiol 33:134-140.

Wang Q, Marcucci F, Cerullo I, Mason C (2016) Ipsilateral and contralateral retinal ganglion cells express distinct genes during decussation at the optic chiasm.eNeuro 3 .

981

982

Williams RW, Bastiani MJ, Lia B, Chalupa LM (1986) Growth cones, dying axons, and developmental fluctuations in the fiber population of the cat's optic nerve. J Comp Neurol 246:32-69.

983 Wong KY (2012) A Retinal Ganglion Cell That Can Signal Irradiance Continuously for 10 Hours. J Neurosci 32:11478-11485.

Young MJ, Lund RD (1994) The anatomical substrates subserving the pupillary light reflex in rats: Origin of the consensual pupillary response. Neuroscience 62:481496.

Young RW (1984) Cell death during differentiation of the retina in the mouse. J Comp Neurol 229:362-373. Hochgeschwender U (2016) Cre-dependent DREADD (Designer Receptors Exclusively Activated by Designer Drugs) mice. Genesis 54:439-446. 


\section{Cdh3-RGC axons (GFP)}

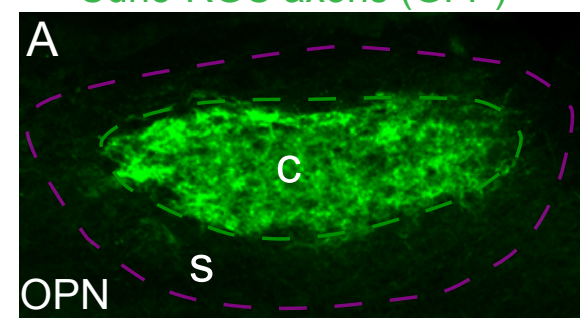

M1 ipRGC axons (Tau-LacZ)

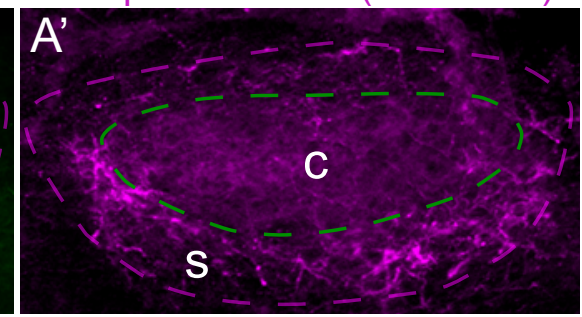

C

3

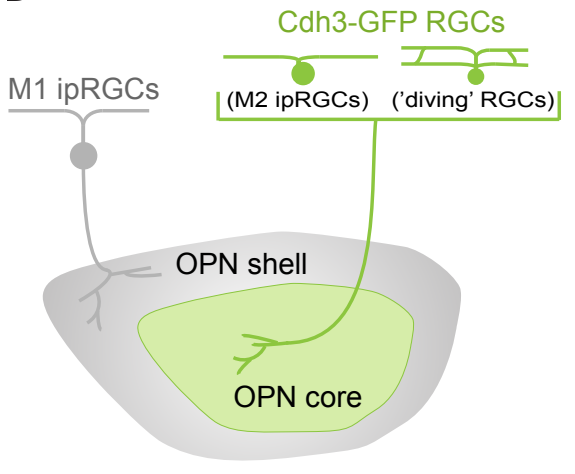

C Distribtion of Cdh3-RGCs (GFP)

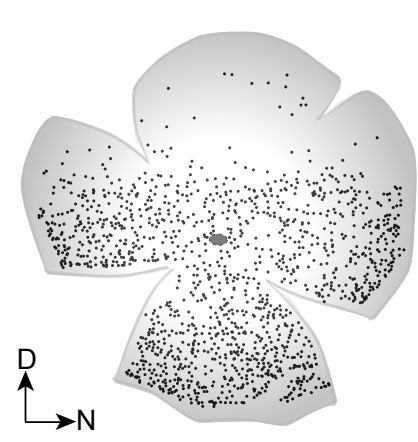

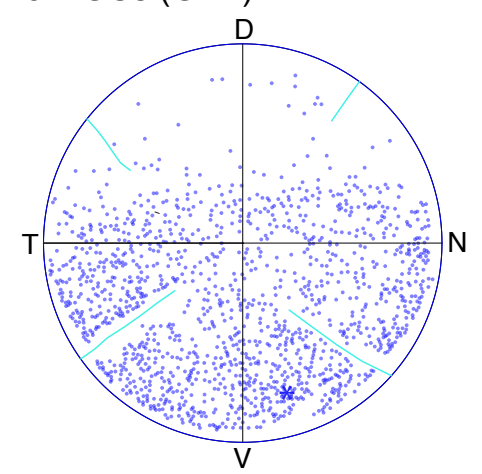

D

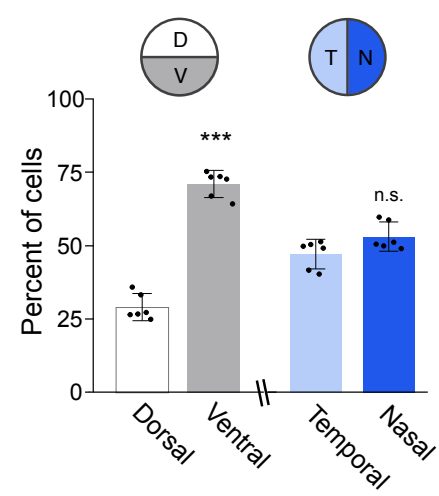

Cdh3-RGCs (GFP)

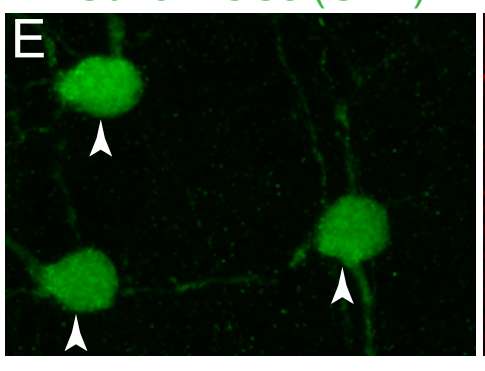

Tbx20

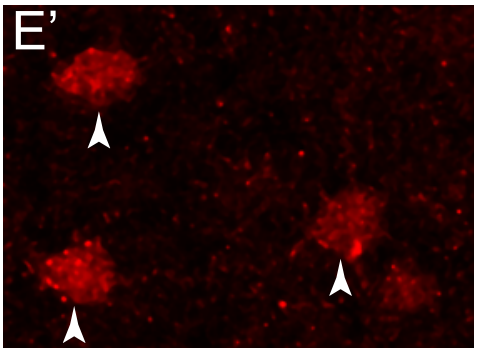

GFP Tbx20 DAPI

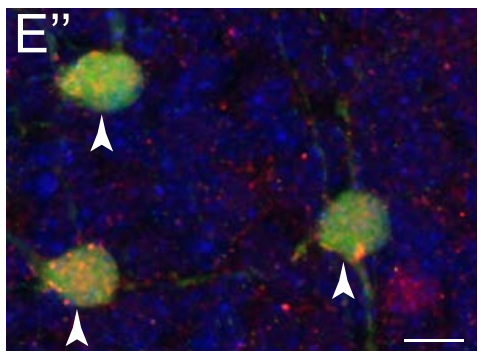

Merge

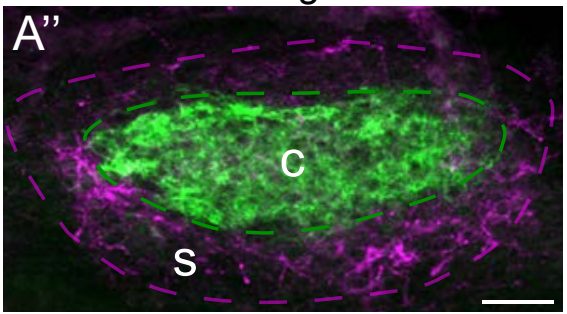

G

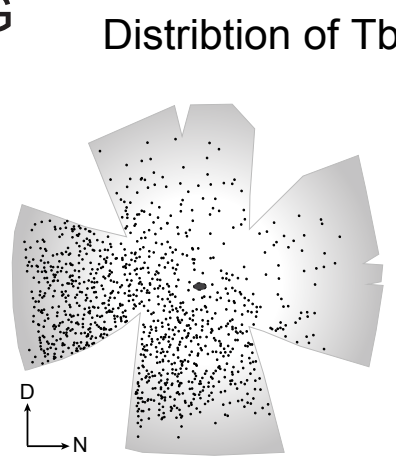

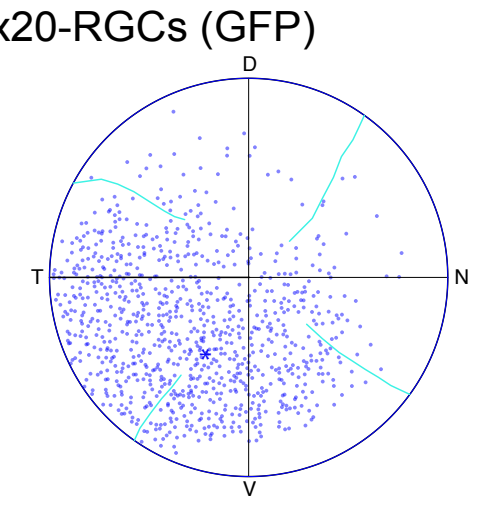
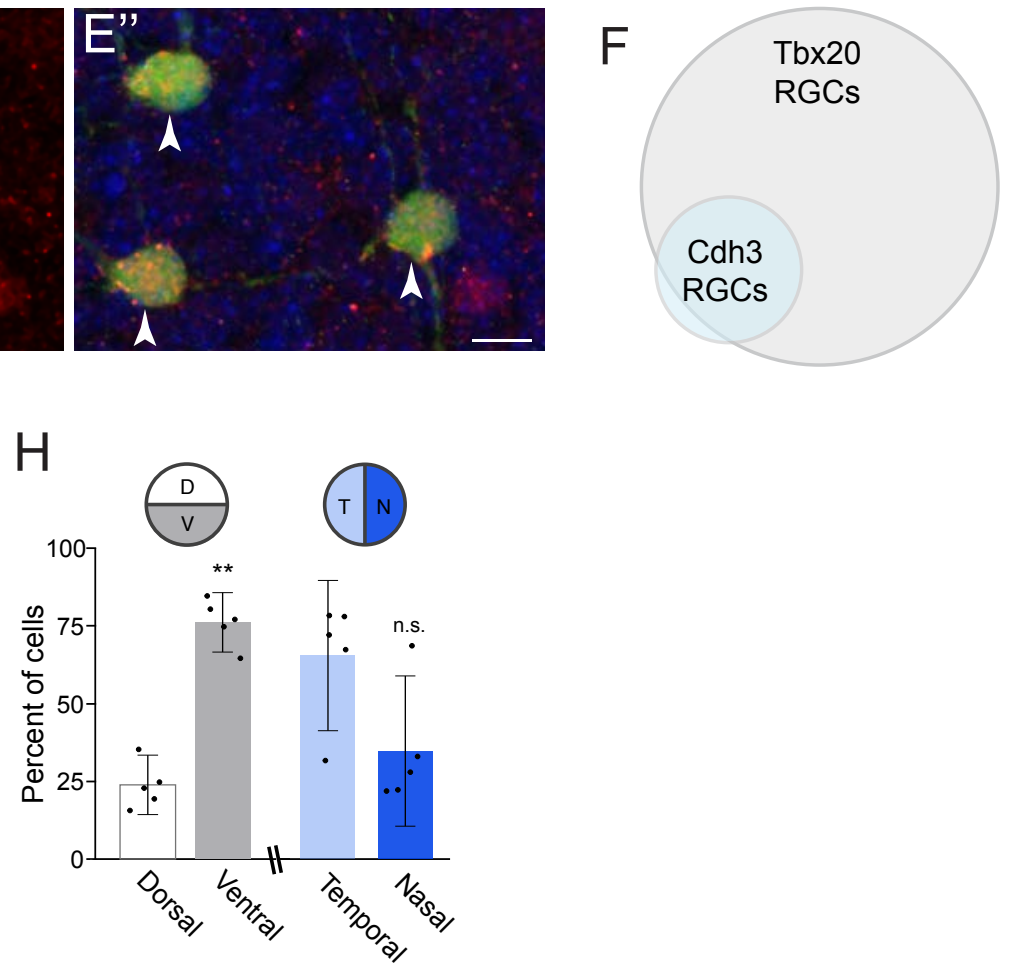

I

Molecular-marker analysis of Tbx20-RGCs
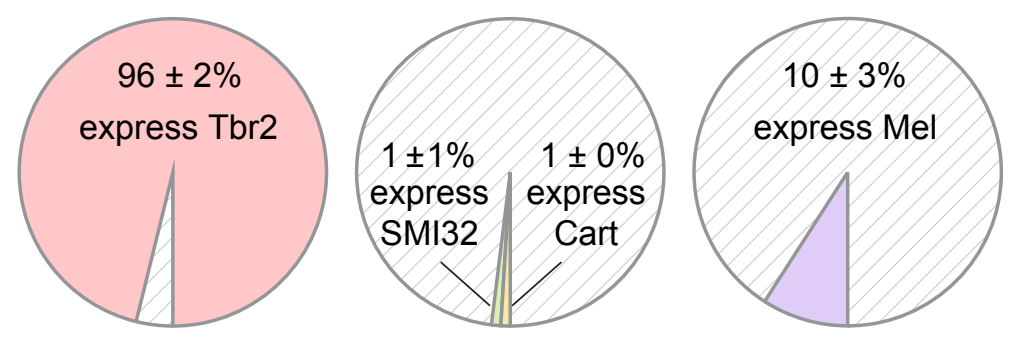


\section{Figure Legends}

1005

Figure 1: Cdh3-RGCs form a unique non-image-forming optic pathway

1007 (A-A') Photomicrographs showing Cdh3-RGC projections (green, GFP) to the OPN core

(c), and M1 ipRGC projections (purple, $\beta$-Gal immunostaining) to the OPN shell (s).

(B) Schematic of the diversity of RGC types innervating the OPN. Cdh3-RGCs include two RGCs subtypes: M2 ipRGCs and 'diving' RGCs.

1013 (C) Retinal flat mount showing the ventral-biased spatial distribution of Cdh3-RGCs

1014 (GFP, black dots, left). Azimuthal equilateral projection of reconstructed retina with

1015 labeled Cdh3 cells (blue circles, right). Blue asterisk is the peak density. Cyan lines

1016 indicate stitching locations from flat mount relieving cuts.

(D) Percent of Cdh3-RGCs (GFP) cells in respective retinal halves: dorsal (29.0 \pm 1.8$)$,

1019 ventral (71.0 \pm 1.8$)$, temporal (47.0 \pm 2.0$)$ and nasal (53.0 \pm 2.0$)$ (Cdh3-GFP RGCs/retina:

$10201005 \pm 74 ; \mathrm{n}=6$ retinas/mice, P20). *** $\mathrm{p}<0.001$; n.s., not significant, $\mathrm{p}>0.05$. Two-tailed

1021 paired Student's t-test. Data shown are Mean \pm SEM.

1022

1023 (E-E') Photomicrograph of Cdh3-RGCs (green, E) co-immunostained with Tbx20 (red,

1024 E') at P4. Cell nuclei are labeled with DAPI (blue, E'). Arrowheads indicate overlap of 1025 GFP and Tbx20.

1027 (F) Venn diagram showing overlap of Cdh3-RGCs and Tbx20-RGCs. (83 $\pm 3 \%$ of Cdh3-

1028 RGCs express Tbx20, 562 GFP (Cdh3-RGCs) cells counted; $38 \pm 1 \%$ of Tbx20 expressing 1029 cells are Cdh3-RGCs, 1242 Tbx20-expressing cells counted; data are Mean \pm SEM; $n=2$ 1030 mice/retinas)

1031

1032 (G) Retinal flat mount showing ventral-biased spatial distribution of Tbx20-RGCs (GFP, 1033 black dots, left). Azimuthal equilateral projection of reconstructed retina with labeled 
1034 Tbx20 cells (blue circles, right). Cyan lines indicate stitching locations from flat mount 1035 relieving cuts.

1036

1037 (H) Percent of Tbx20-GFP cells in respective retinal halves: dorsal (23.9 \pm 3.1), ventral 1038 (76.1 \pm 3.1$)$, temporal (65.4 \pm 7.9$)$ and nasal (34.6 \pm 7.9$)($ Tbx20-RGCs/retina: $863 \pm 54$; $1039 \mathrm{n}=5$ retinas/mice, P20). *** $\mathrm{p}<0.01$; n.s., not significant, $\mathrm{p}>0.05$. Two-tailed paired 1040 Student's t-test. Data shown are Mean \pm SEM.

1041

1042 (I) Quantification of expression of RGC type-specific markers by Tbx20-RGCs. Data 1043 shown are Mean \pm SEM. (Tbr2: $\mathrm{n}=3$ mice/retinas, 374 Tbx20-GFP cells counted; Cart: $\mathrm{n}=2$ 1044 mice/retinas, 282 Tbx20-GFP cells counted; SMI32: $\mathrm{n}=2$ mice/retinas, 184 Tbx20-GFP 1045 cells; Melanopsin: $\mathrm{n}=4$ mice/retinas, 693 Tbx20-GFP cells)

1046

1047 See also Figure S1 and Figure S2.

1048

1049 D: dorsal; N: nasal; V: ventral; T: temporal. Scale bar: $100 \mu \mathrm{m}$ (A’’), $10 \mu \mathrm{m}(\mathrm{E}$ ’')

1050

1051

1052

1053

1054

1055

1056

1057

1058

1059

1060

1061

1062 


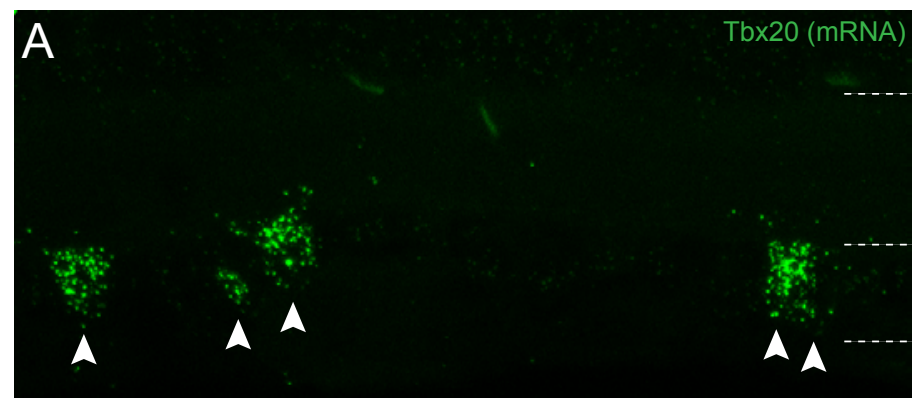

B

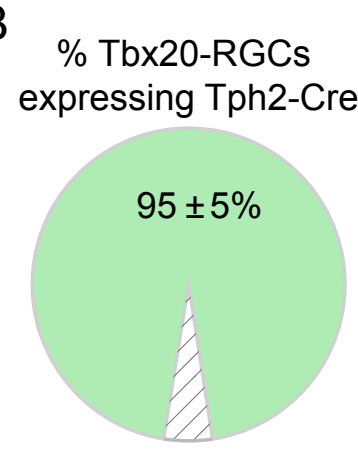

\section{Cdh3-RGCs (GFP)}

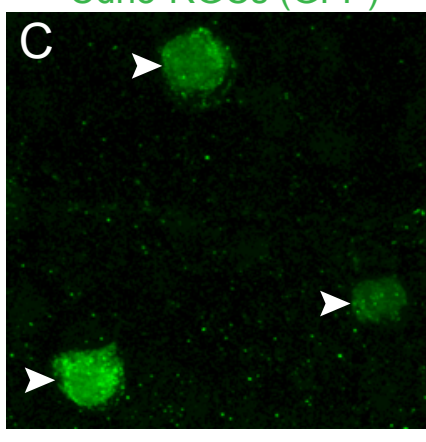

Control
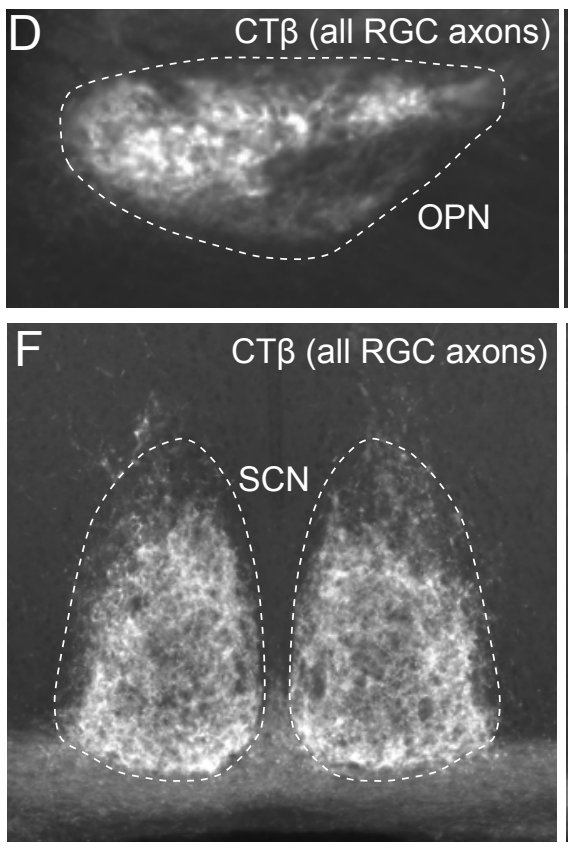

$\mathrm{H}$

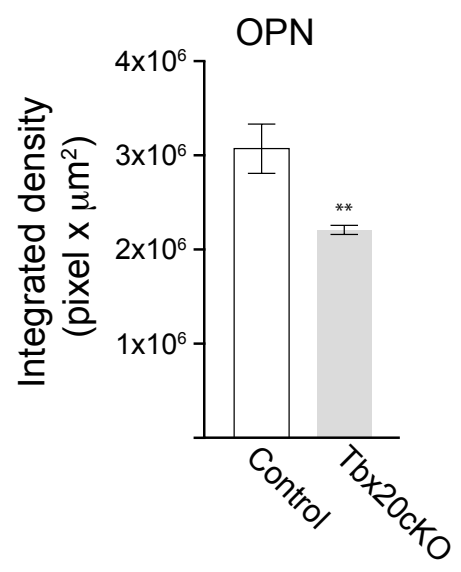

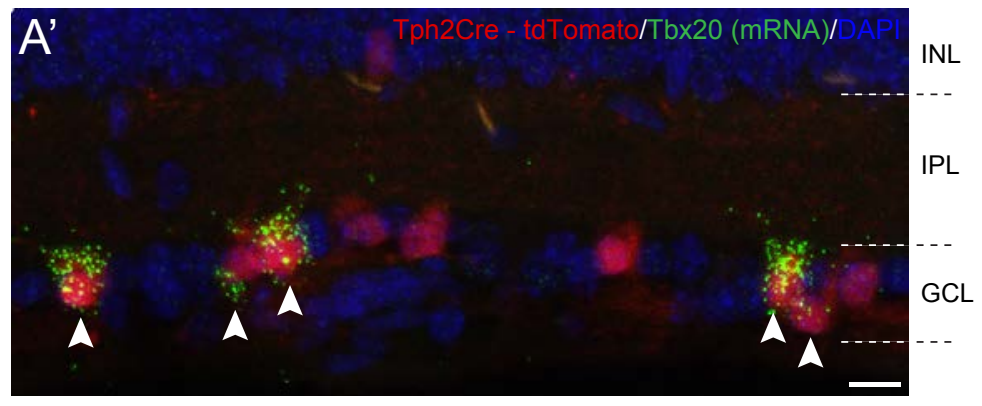

Tph2Cre - tdTomato
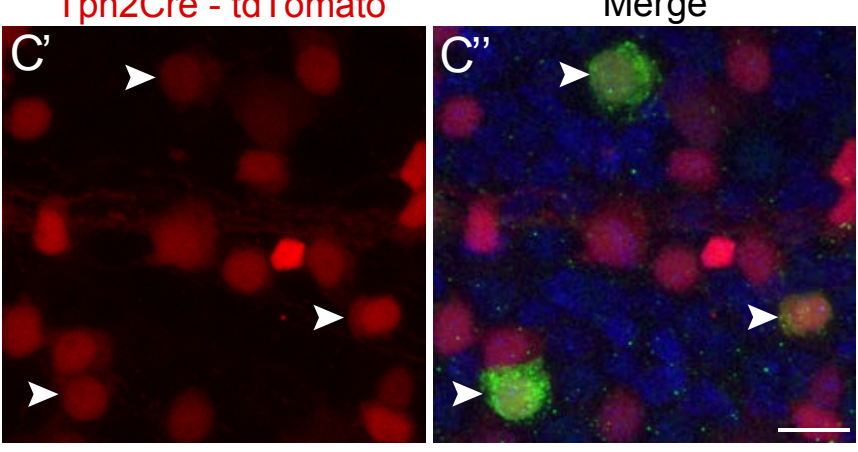

Tbx20cKO
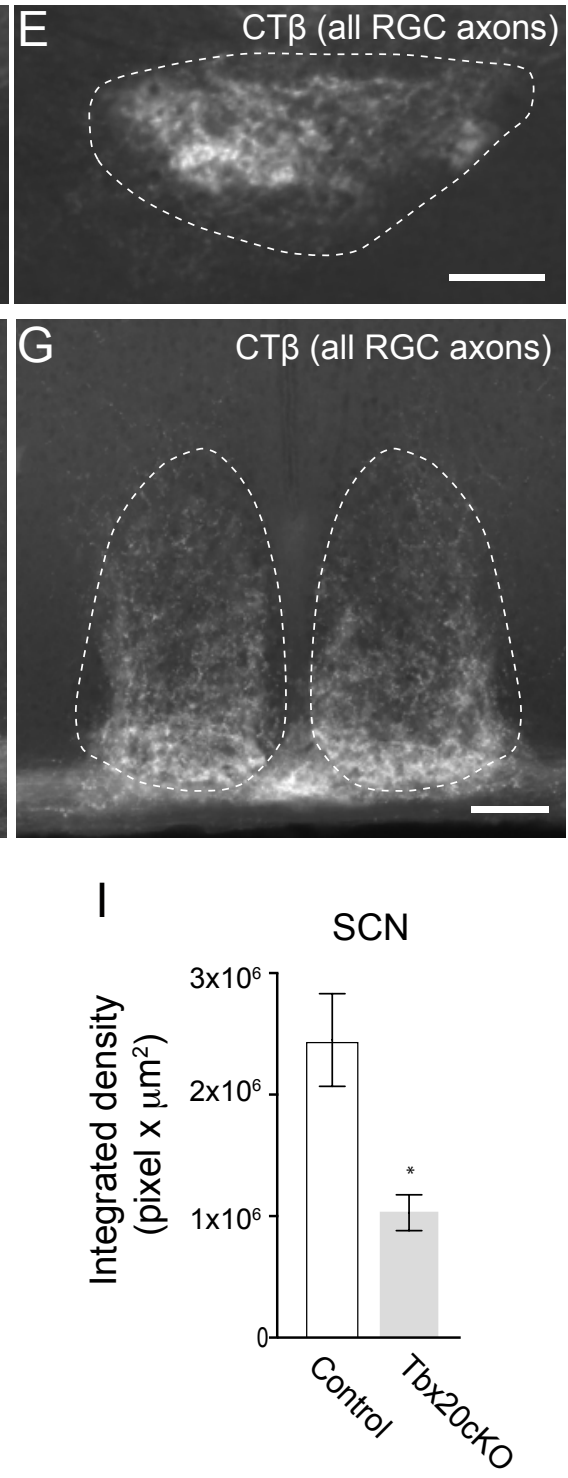


\section{Figure 2: Loss of Tbx20 results in reduced retinal innervation of non-image-forming} 1065 targets

1066 (A-A') Photomicrographs showing cells expressingTbx20 mRNA (green, A) also express 1067 Cre recombinase (visualized by tdTomato reporter, endogenous signal, red A') at P19. 1068 Nuclei are labeled with DAPI (blue, A'). Arrowheads indicate overlap of Tbx20 mRNA 1069 and tdTomato (Cre).

(B) Quantification of expression of Cre recombinase (tdTomato reporter) by Tbx20-RGCs.

1072 Data shown is Mean \pm SEM. ( $\mathrm{n}=2$ mice/retinas, 153 Tbx20 cells)

1073

1074 (C-C'") Photomicrographs showing Cdh3-RGCs (green, C) overlap with Cre recombinase 1075 (visualized by tdTomato reporter, red, C') expressing cells. Nuclei are labeled with DAPI 1076 (blue, C'’) Arrowheads indicate overlap of GFP and tdTomato (Cre).

1078 (D-G) Example photomicrographs showing RGC innervation (visualized by CT $\beta$ labeling) 1079 of OPN and SCN in control and Tbx20 cKO mice.

1081 (H, I) Quantification of density of retinofugal innervation in OPN (D, E) (Control Mean \pm 1082 SEM: $3.1 \times 10^{6} \pm 2.6 \times 10^{5}$ pixel x $\mu \mathrm{m}^{2}, \mathrm{n}=6$ mice; Tbx20 cKO Mean \pm SEM: $2.2 \times 10^{6} \pm$ $10834.8 \times 10^{4}$ pixel $x \mu \mathrm{m}^{2}, \mathrm{n}=6$ mice) and SCN (F, G) (Control Mean \pm SEM: $2.5 \times 10^{6} \pm 3.8 \times 10^{5}$ 1084 pixel $x \mu \mathrm{m}^{2}, \mathrm{n}=6$ mice; Tbx20 cKO Mean \pm SEM: $1.0 \times 10^{6} \pm 1.5 \times 10^{5}$ pixel $\mathrm{x} \mu \mathrm{m}^{2}, \mathrm{n}=4$ 1085 mice) in control and Tbx20 cKO mice at P20.

1086

1087 See also Figure S3 and Figure S4.

1088

1089 GCL: ganglion cell layer; IPL: inner plexiform layer; INL: inner nuclear layer; OPN: 1090 olivary pretectal nucleus; SCN: suprachiasmatic nucleus.

1091 Scale bar: $10 \mu \mathrm{m}\left(\mathrm{A} ", \mathrm{C}\right.$ ") $, 100 \mu \mathrm{m}(\mathrm{E}, \mathrm{G}) .{ }^{*} \mathrm{p}<0.05, * * \mathrm{p}<0.01$. Student's t-test. 
Cdh3-RGCs Tbx20 cKO
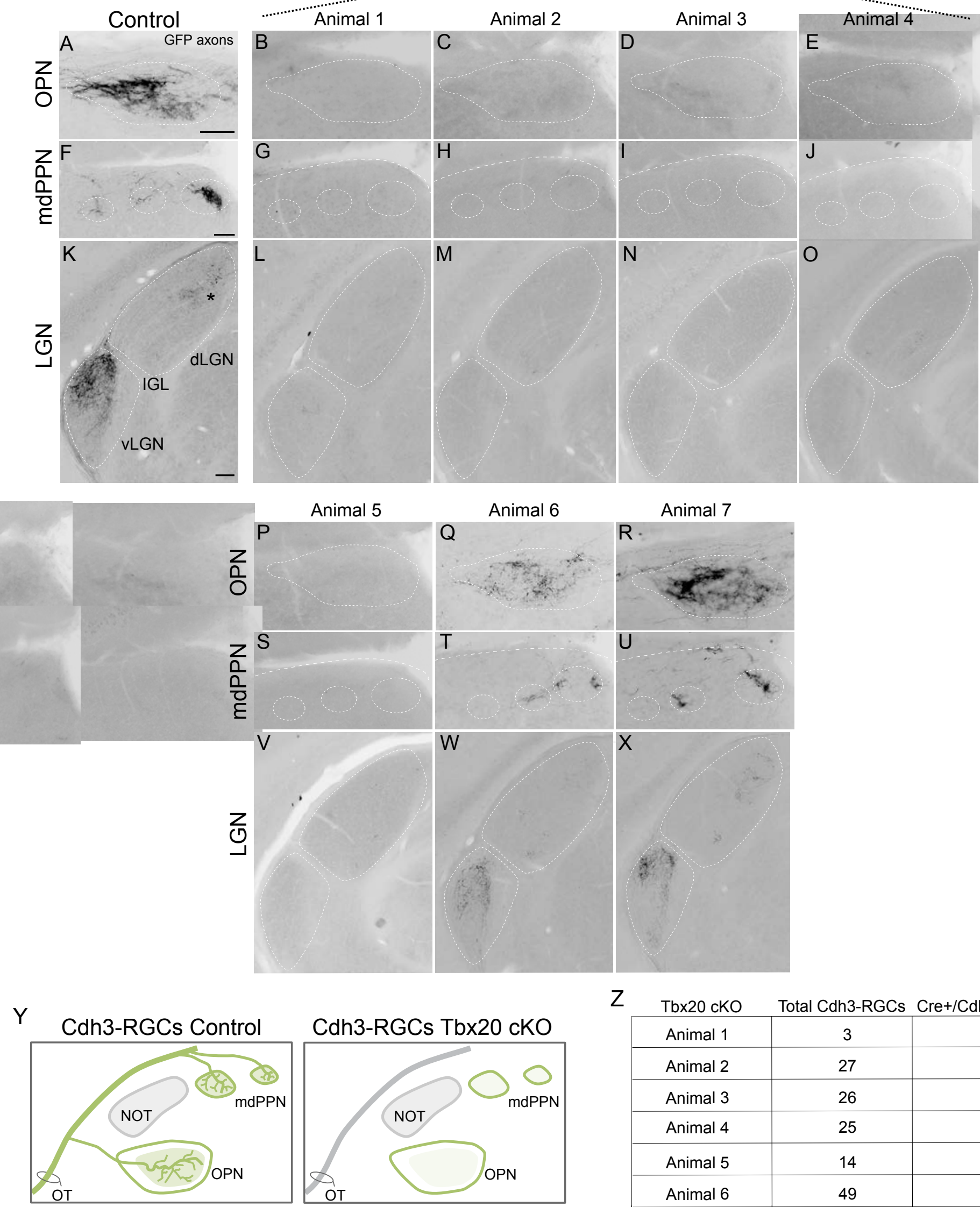

Z

\begin{tabular}{|c|c|c|}
\multicolumn{1}{|c|}{ Tbx20 cKO } & Total Cdh3-RGCs & Cre+/Cdh3-RGCs \\
\hline Animal 1 & 3 & 1 \\
\hline Animal 2 & 27 & 1 \\
\hline Animal 3 & 26 & 0 \\
\hline Animal 4 & 25 & 0 \\
\hline Animal 5 & 14 & 0 \\
\hline Animal 6 & 49 & 3 \\
\hline Animal 7 & 54 & 7 \\
\hline
\end{tabular}


1095 Figure 3: Conditional deletion of Tbx20, a transcription factor highly enriched in 1096 Cdh3-RGCs, results in loss of Cdh3-RGC projection pathway

1097 (A, F, K) Photomicrographs showing innervation of OPN and mdPPN by Cdh3-RGC axons

1098 (black) in control animals. In the visual thalamus, Cdh3-RGC axons selectively terminate

1099 in the vLGN and sparsely innervate the dorsal-medial half of the dLGN (asterisk).

1100

1101 (B-E, G-J, L-O, P-X) Photomicrographs demonstrating the variability in the lack of 1102 innervation of OPN, mdPPN and LGN by Cdh3-RGC axons in Tbx20 cKO mice.

1103 (Y) Schematics depicting typical outcome of Cdh3-RGC innervation of their normal targets 1104 in control and Tbx20 cKO mice.

1105

1106 (Z) Quantification of total number of Cdh3-RGCs and fraction of Cre expressing Cdh3-

1107 RGCs remaining in Tbx20 cKO mice. The level of OPN, mdPPN and LGN innervation by

1108 Cdh3-RGC axons in Tbx20 cKO mice is strongly correlated with the number of spared 1109 (Cre negative) Cdh3-RGCs.

1110 OPN: olivary pretectal nucleus; mdPPN: medial division of the posterior pretectal nucleus;

1111 dLGN: dorsal lateral geniculate nucleus; vLGN: ventral LGN; IGL: intergeniculate leaflet.

1112 Scale bar: $100 \mu \mathrm{m}(\mathrm{A}, \mathrm{F}, \mathrm{K})$.

1113

1114

1115

1116

1117

1118

1119

1120

1121

1122

1123

1124 
bioRxiv preprint doi: https://doi.org/10.1101/500868; this version posted December 19, 2018. The copyright holder for this preprint (which was Figuree 4

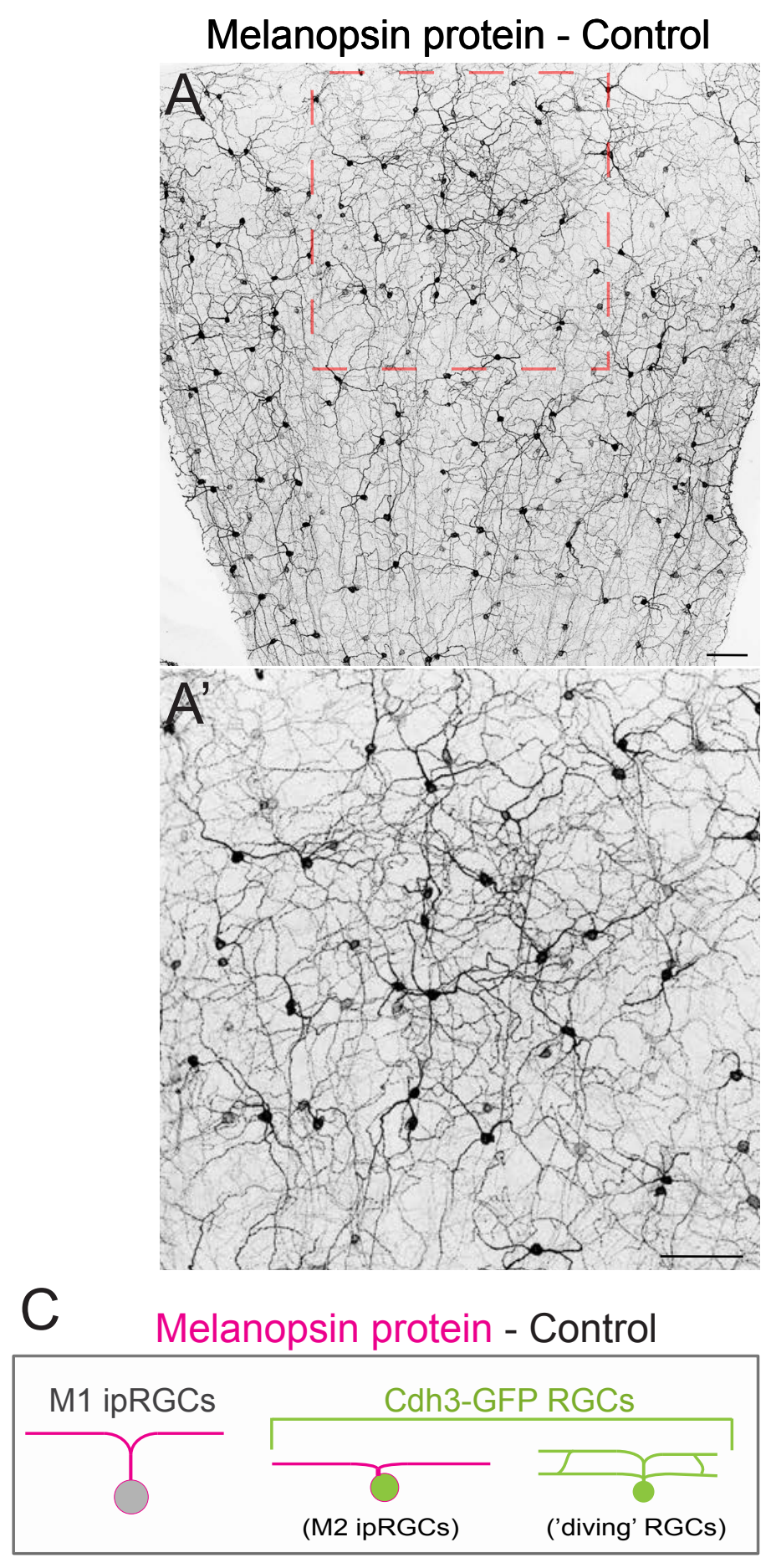

C under aCC-BY-ND 4.0 International license.
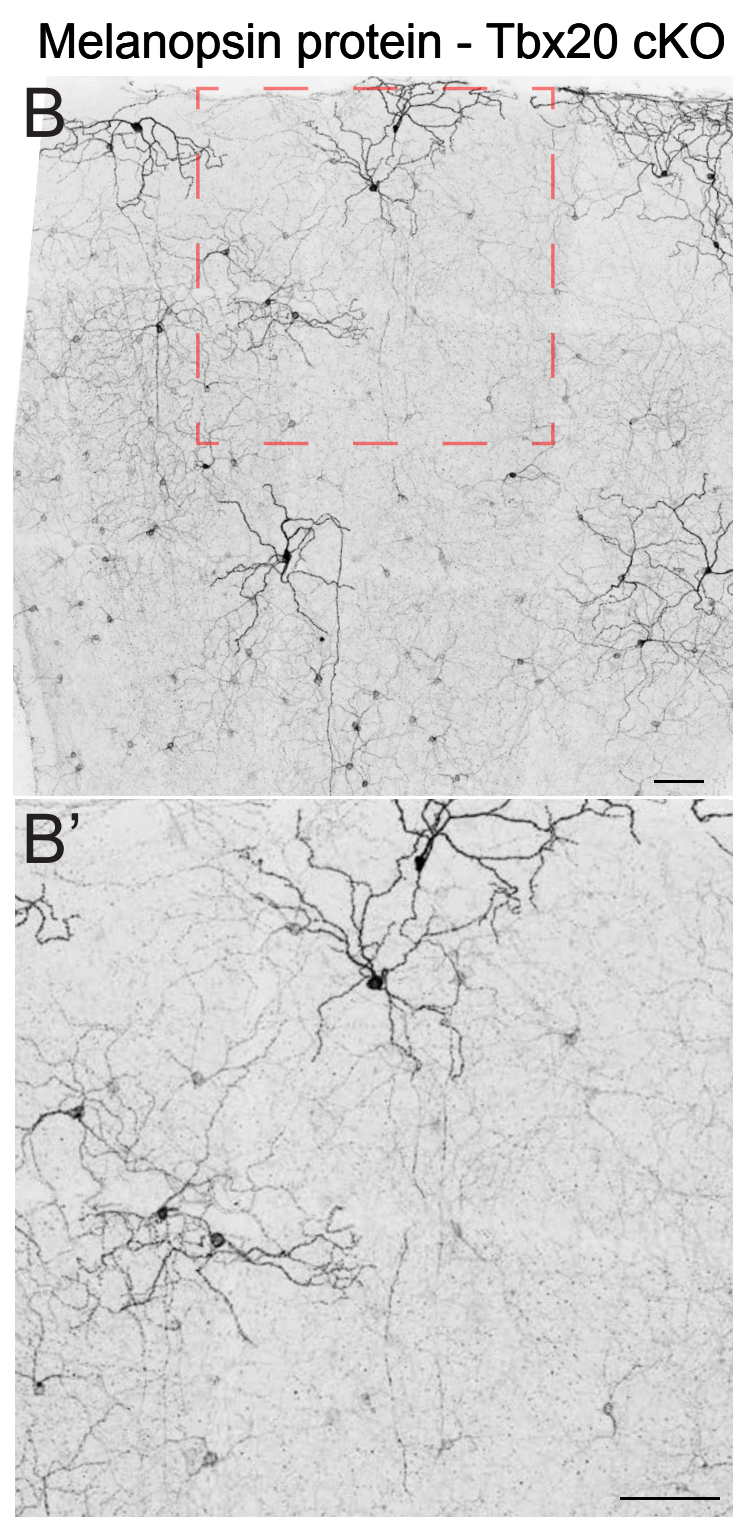

Melanopsin protein -Tbx20 cKO

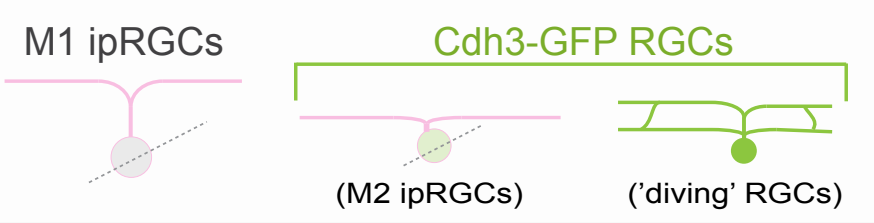


1125 Figure 4: The mosaic pattern of intrinsically photosensitive RGCs that express high 1126 levels of melanopsin protein is disrupted in Tbx20 conditional mutants

1127 (A-B') Photomicrographs showing melanopsin labeling in control (A, A') and Tbx20 cKO

1128 (B, B') retinas.

1129

1130 (C) Schematics depicting reduced melanopsin-expressing RGCs (M1 ipRGCs) in control 1131 and $\mathrm{Tbx} 20 \mathrm{cKO}$ retinas.

1132

1133 Scale bars: $100 \mu \mathrm{m}\left(\mathrm{A}-\mathrm{B}^{\prime}\right)$.

1134

1135

1136

1137

1138

1139

1140

1141

1142

1143

1144

1145

1146

1147

1148

1149

1150

1151

1152

1153

1154

1155 
bioRxiv preprint doi: https://doi.org/10.1101/500868; this version posted December 19, 2018. The copyright holder for this preprint (which was not certified by peer review) is the author/funder, who has granted bioRxiv a license to display the preprint in perpetuity. It is made available

\section{Figure 5} under aCC-BY-ND 4.0 International license.

M1 ipRGCs - Control

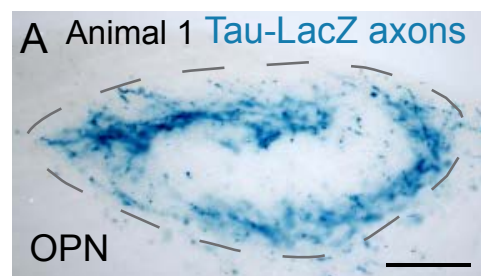

$\mathrm{E}$

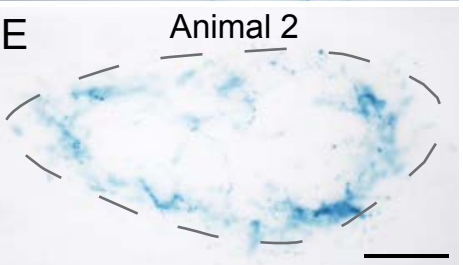

M1 ipRGCs - Control

I Animal 1 Tau-LacZ axons

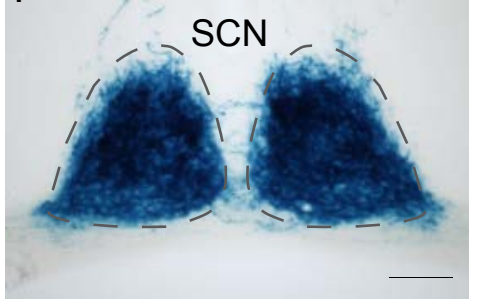

L

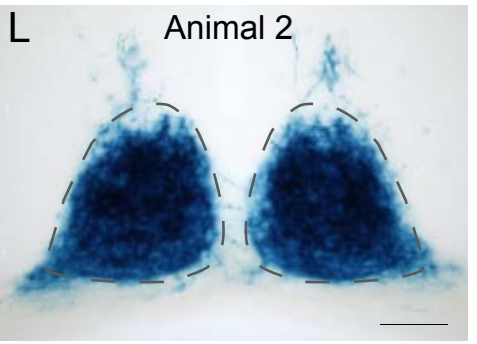

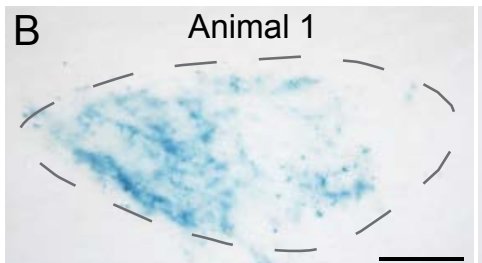

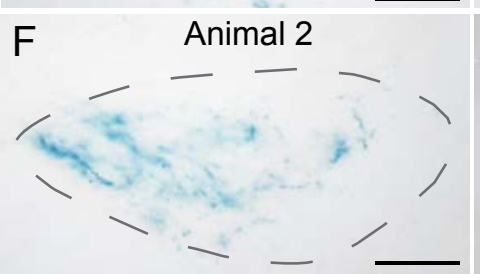

M1 ipRGCs -Tbx20 cKO

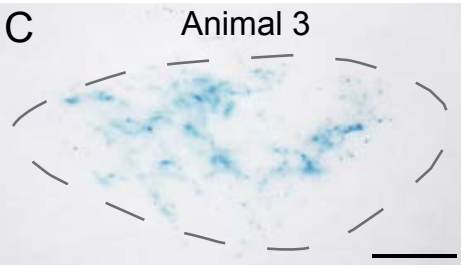

D

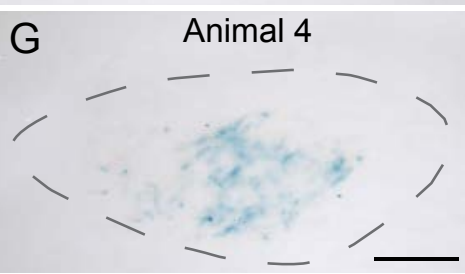

M1 ipRGCs - Tbx20 cKO
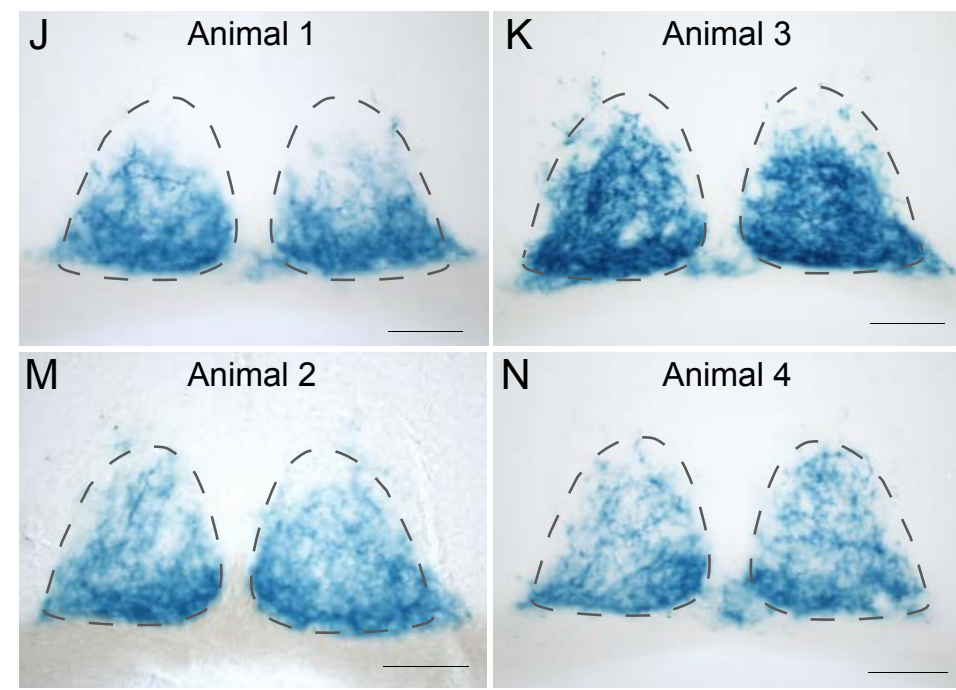

$\mathrm{N}$

Animal 4

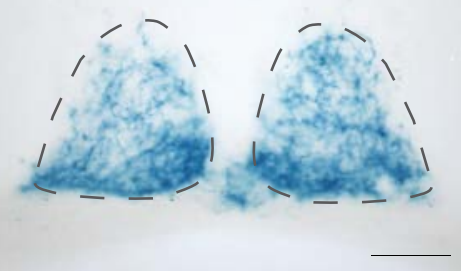

\section{Q}
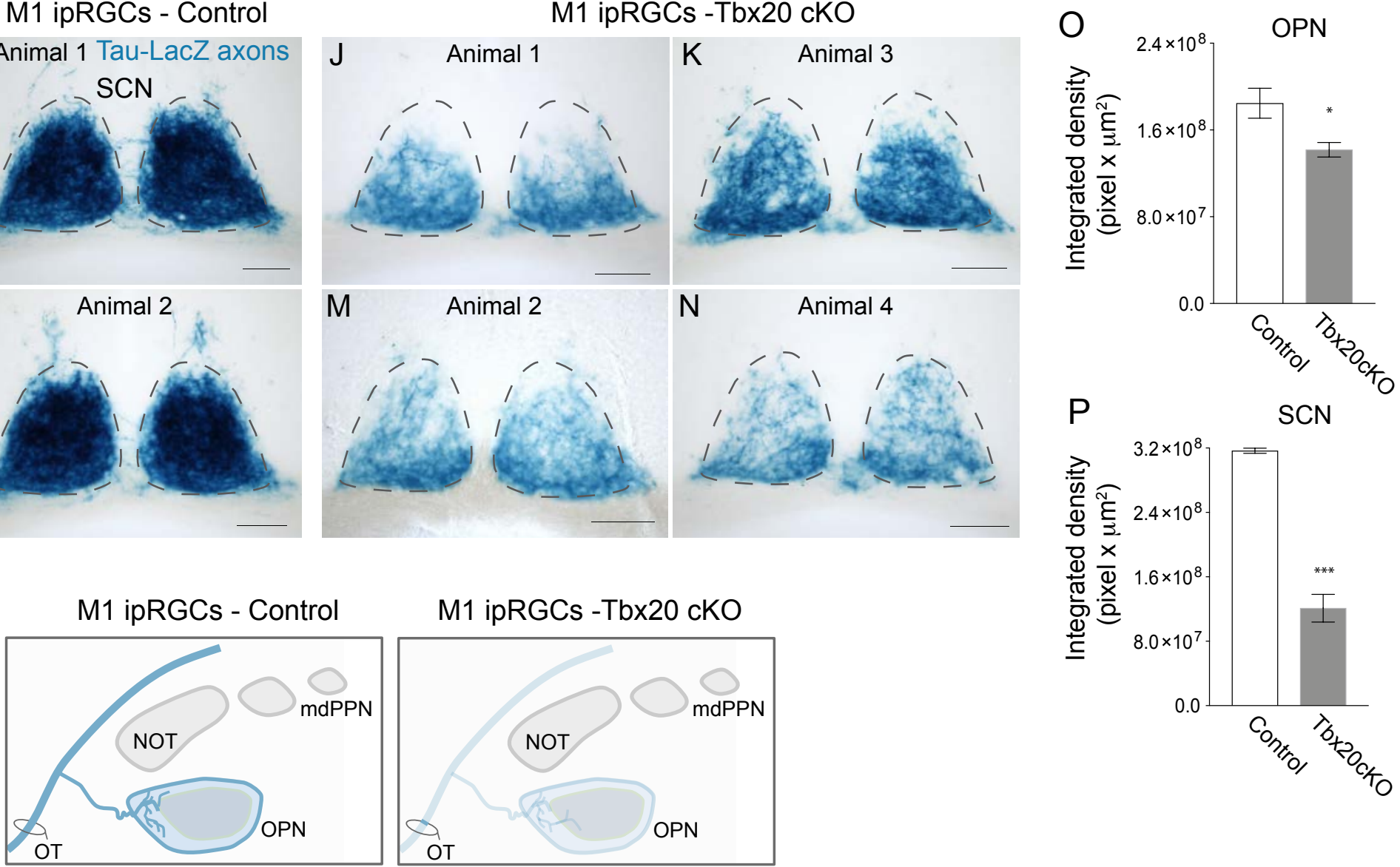
1156 Figure 5: Loss of Tbx20 results in reduced M1 ipRGCs axonal innervation of the OPN 1157 and the SCN

1158 (A-H) Photomicrographs showing innervation of M1 ipRGCs (visualized by $\beta$-gal staining, 1159 blue) in OPN of control (A, E) and Tbx20 cKO mice (B-D, F-H).

1161 (I-N) Photomicrographs showing innervation of M1 ipRGCs (visualized by $\beta$-gal staining, 1162 blue) in SCN of control (I, L) and Tbx20 cKO mice (J, K, M, L).

1163

1164 (O) Quantification of density of M1 ipRGC innervation in OPN ( $\beta$-gal staining density 1165 Control Mean \pm SEM: $1.85 \times 10^{8} \pm 1.38 \times 10^{7}$ pixel $\times \mu \mathrm{m}^{2}, \mathrm{n}=6$ mice; Tbx20 cKO Mean \pm 1166 SEM: $1.42 \times 10^{8} \pm 6.74 \times 10^{6}$ pixel $\times \mu \mathrm{m}^{2}, \mathrm{n}=6$ mice) .

1168 (P) Quantification of density of M1 ipRGC innervation in SCN ( $\beta$-gal staining density 1169 Mean \pm SEM: Control: $3.17 \times 10^{8} \pm 3.19 \times 10^{6}$ pixel $\times \mu \mathrm{m}^{2}, \mathrm{n}=6$ mice; Tbx $20 \mathrm{cKO}: 1.21 \times 10^{8}$ $1170 \pm 1.72 \times 10^{7}$ pixel $\times \mu \mathrm{m}^{2}, \mathrm{n}=6$ mice) .

(Q) Schematics depicting typical outcomes of M1 ipRGCs innervation of the OPN in 1173 control and Tbx20 cKO mice.

1174

1175 OPN: olivary pretectal nucleus; SCN: suprachiasmatic nucleus.

1176 $* \mathrm{p}<0.05, * * * \mathrm{p}<0.001$, Student's t-test. Scale bars: $100 \mu \mathrm{m}(\mathrm{A}-\mathrm{N})$.

1177

1178

1179

1180

1181

1182

1183

1184

1185

1186 
bioRxiv preprint doi: https://doi.org/10.1101/500868; this version posted December 19, 2018. The copyright holder for this preprint (which was not certified by peer review) is the author/funder, who has granted bioRxiv a license to display the preprint in perpetuity. It is made available under aCC-BY-ND 4.0 International license.

\section{Figure 6}
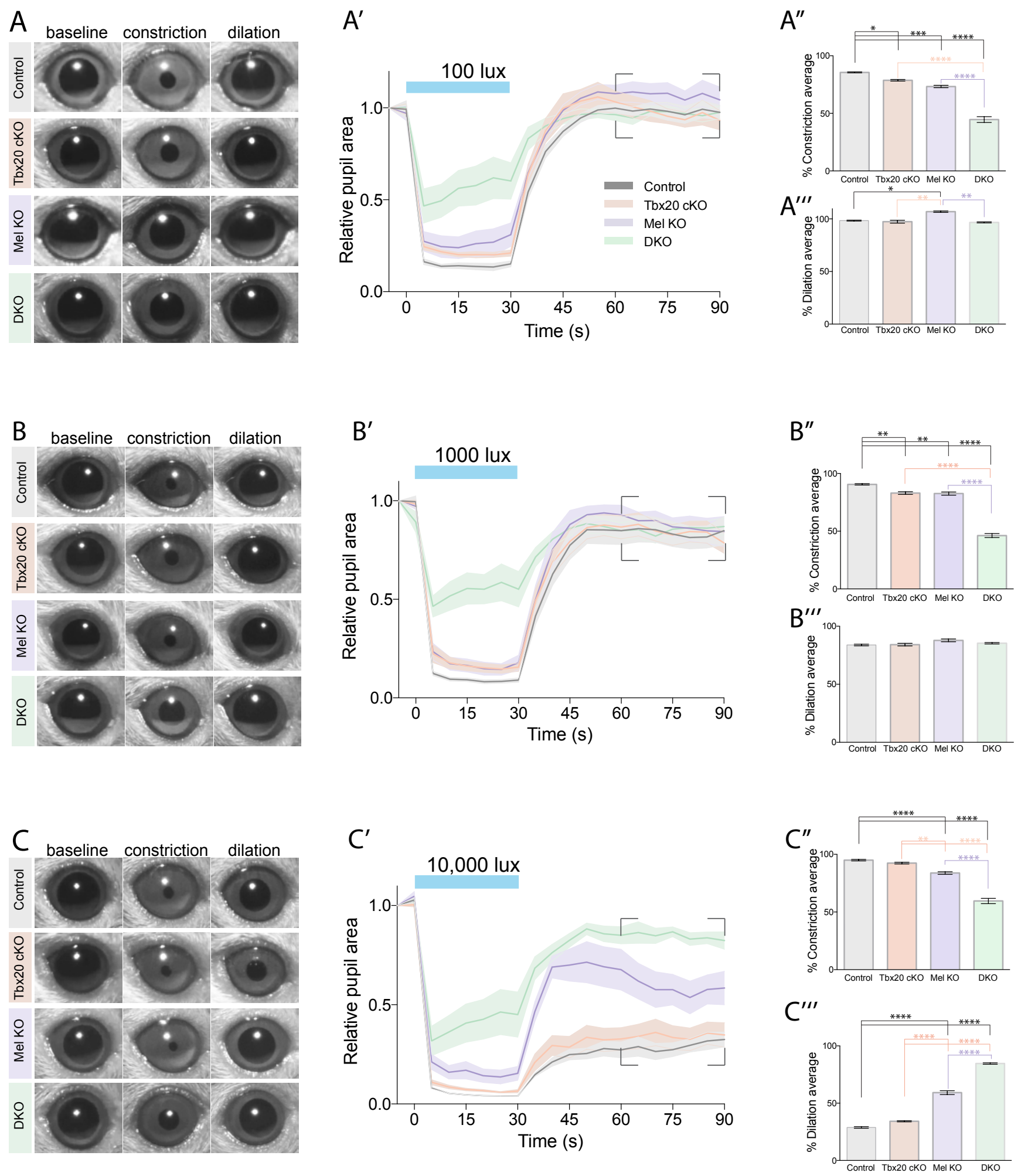


\section{Figure 6: Loss of Tbx20-RGCs minimally impacts the pupillary light reflex}

1188 (A, B, C) Representative images of control, Tbx20 cKO, Melanopsin (Mel) KO and 1189 Tbx20/Melanopsin double KO (dKO) mice tested with a 100 lux (A), 1,000 lux (B) and

1190 10,000 lux (C) light stimulus at different stages of PLR: baseline (0 s), constriction (30 s), 1191 and dilation (60 s).

1192

1193 (A', B', C') PLR traces at $100 \operatorname{lux}\left(\mathrm{A}^{\prime}\right)$, 1,000 lux (B') and 10,000 lux (C').

1194 Mean \pm SEM (shaded).

1195

1196 (A") Average \% pupil constriction in control, Tbx20 cKO, Mel KO, and dKO mice during 1197100 lux (Control: Mean \pm SEM: $85.57 \pm$ 0.45\%; Tbx20 cKO: Mean \pm SEM: $78.72 \pm 0.70 \%$;

1198 Mel KO: Mean \pm SEM: $73.32 \pm 1.04 \%$; dKO: Mean \pm SEM: $44.61 \pm 2.48 \%$ ).

1200 (B”) Average \% pupil constriction in control, Tbx20 cKO, Mel KO, and dKO mice during 1201 1,000 lux (Control: Mean \pm SEM: $90.55 \pm$ 0.62\%; Tbx20 cKO: Mean \pm SEM: $83.03 \pm$ 1202 1.17\%; Mel KO: Mean \pm SEM: $82.58 \pm 1.34 \%$; dKO: Mean \pm SEM: $46.25 \pm 1.71 \%$ ).

1204 (C') Average \% pupil constriction in control, Tbx20 cKO, Mel KO, and dKO mice during 1205 10,000 lux (Control: Mean \pm SEM: $91.90 \pm$ 0.64\%; Tbx20 cKO: Mean \pm SEM: $89.06 \pm$ 1206 0.75\%; Mel KO: Mean \pm SEM: $78.82 \pm$ 1.1\%; dKO: Mean \pm SEM: $53.65 \pm 2.27 \%$ ).

1208 (A"') Average \% pupil dilation in control, Tbx20 cKO, Mel KO and dKO mice between 1209 30-60s post-illumination boxed region in A' at 100 lux (A"') (Control: Mean \pm SEM: 98.38 $1210 \pm$ 0.46\%; Tbx20 cKO: Mean \pm SEM: $97.38 \pm$ 1.39\%; Mel KO: Mean \pm SEM: $106.9 \pm$ 1211 0.68\%; dKO: Mean \pm SEM: $96.71 \pm 0.48 \%)$.

1212 (B"') Average \% pupil dilation in control, Tbx20 cKO, Mel KO and dKO mice between 1213 30-60s post-illumination boxed region in B' in 1,000 lux (B"') (Control: Mean \pm SEM: $121483.85 \pm$ 0.69\%; Tbx20 cKO: Mean \pm SEM: 84.16 \pm 1.15\%; Mel KO: Mean \pm SEM: 87.81 $1215 \pm 1.19 \%$; dKO: Mean \pm SEM: $85.34 \pm 0.59 \%)$.

1216 
1217 (C") Average \% pupil dilation in control, Tbx20 cKO, Mel KO and dKO mice between

1218 30-60s post-illumination boxed region in C' in 10,000 lux (C"') (Control: Mean \pm SEM:

$121928.83 \pm 0.71 \%$; Tbx20 cKO: Mean \pm SEM: $34.21 \pm 0.51 \%$; Mel KO: Mean \pm SEM: 59.22

$1220 \pm 1.68 \%$, dKO: Mean \pm SEM: $84.59 \pm 0.62 \%)$.

1221

1222 Control: $\mathrm{n}=9$ mice; Tbx20 cKO: $\mathrm{n}=10$ mice; Mel KO: $\mathrm{n}=7$ mice; dKO: $\mathrm{n}=6$ mice for 1223 all conditions.

$1224 * \mathrm{p}<0.05, * * \mathrm{p}<0.01, * * * \mathrm{p}<0.001, * * * * \mathrm{p}<0.0001$. Two-way ANOVA with Bonferroni 1225 post-hoc test.

1226

1227

1228

1229

1230

1231

1232

1233

1234

1235

1236

1237

1238

1239

1240

1241

1242

1243

1244

1245

1246

1247 

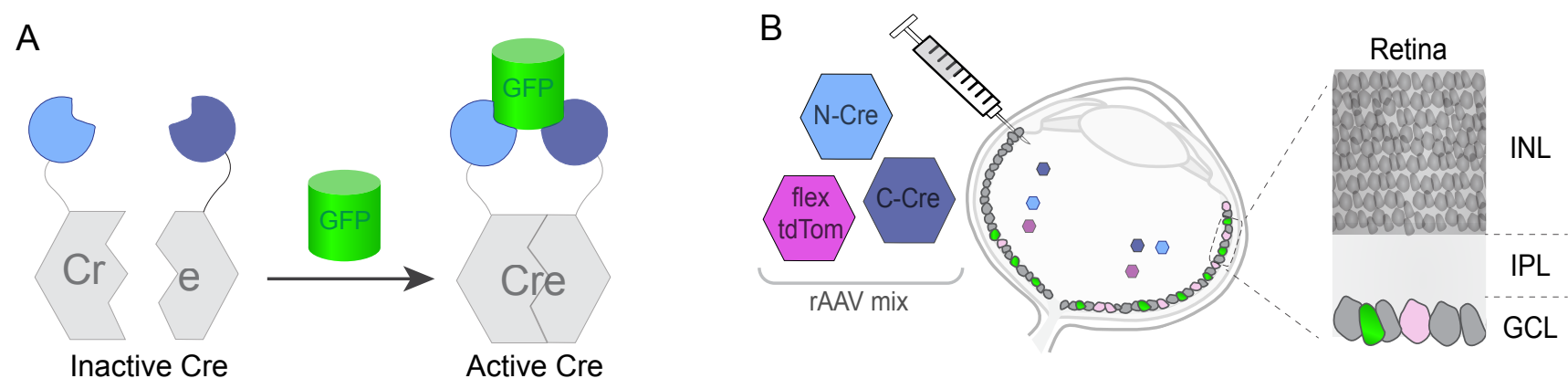

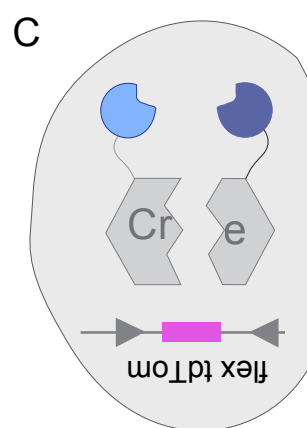

Inactive Cre

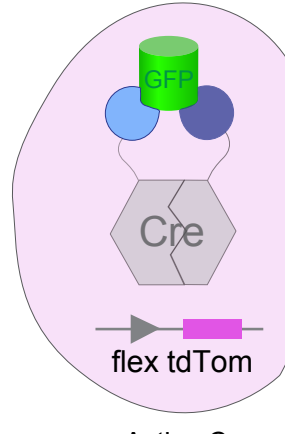

Active Cre
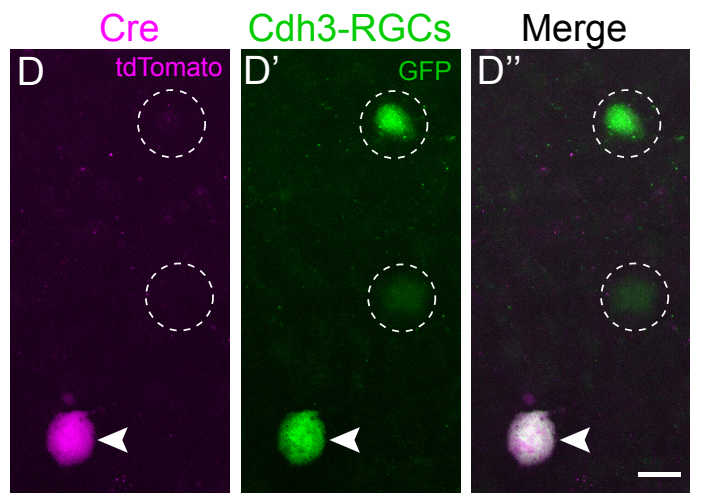
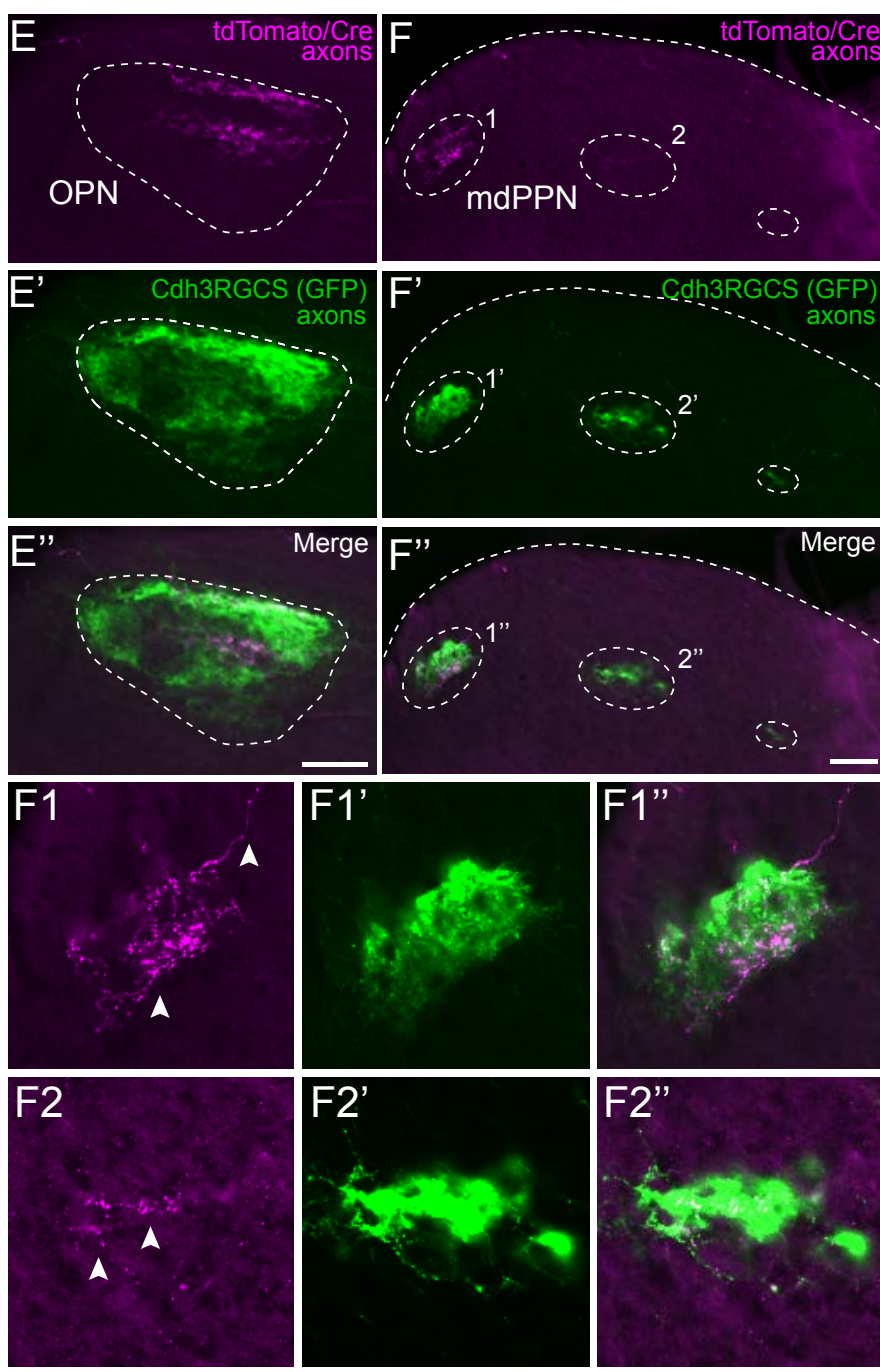

G

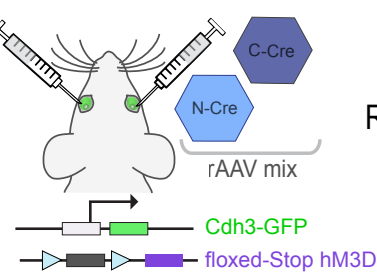

$\mathrm{H}$

AAV injections Dark record CNO record Round 1 Round 2 adapt (pre-CNO) (i.p.) (post-CN(

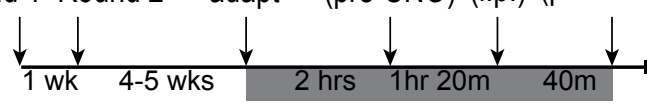

I Cdh3GFP+; flox-Stop hM3D; Cre-

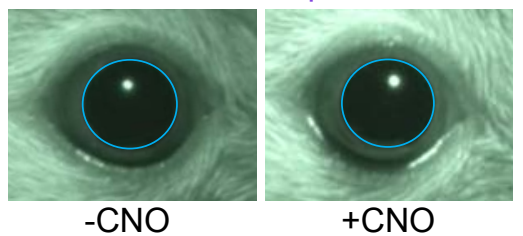

Cdh3GFP+; hM3D+; Cre+

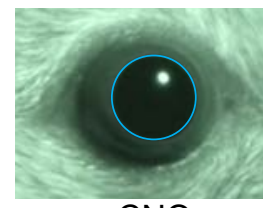

$-\mathrm{CNO}$

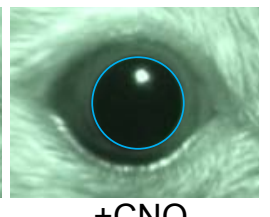

$J$

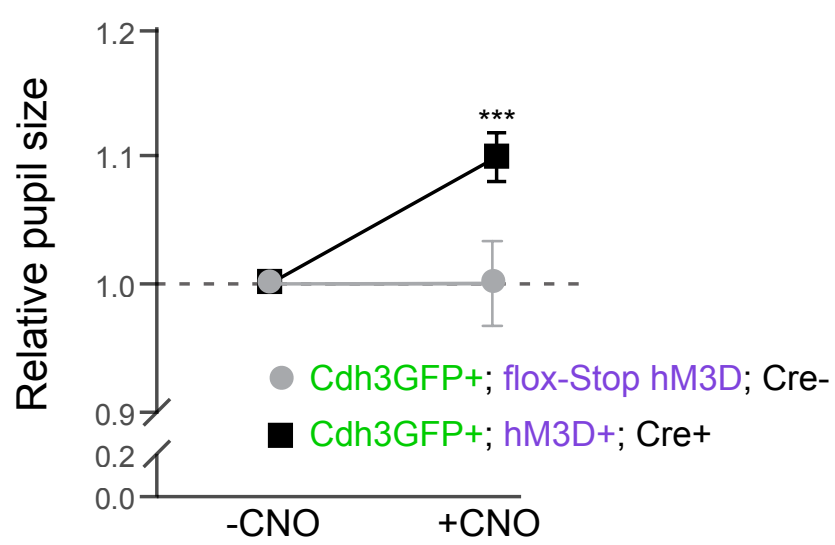




\section{Figure 7: Activation of Cdh3-RGCs increases pupil dilation}

1249 (A) Schematic of Cre-DOG (dependent on GFP) system. Complementary split components

1250 of Cre bind to GFP thereby inducing Cre recombinase mediate activity.

1252 (B) Schematic of ocular injection of viruses encoding the Cre-DOG system (N-Cre and C-

1253 Cre) and a Cre-activity reporter (flex-TdTomato). Close up schematic shows resultant

1254 tdTomato ${ }^{+}$RGCs (pink, Cre active), $\mathrm{GFP}^{+}$(green) and GFP' (grey) RGCs.

1255

1256 (C) TdTomato reporter is expressed only when a $\mathrm{GFP}^{+} \mathrm{RGCs}$ are infected by and expresses

1257 all three viral components: N-Cre, C-Cre and flex-Tdomato (pink). GFP ${ }^{+}$neurons (green)

1258 that do not express both split-Cre components do express the tdTomato. GFP- neurons

1259 (grey) cannot combine the split-Cre system to induce Cre activity.

1261 (D-D”) Photomicrographs showing Cdh3-RGC-specific expression of Cre recombinase 1262 (visualized via tdTomato reporter). Arrowheads indicate tdTomato ${ }^{+}$RGCs that are also 1263 Cdh3-RGCs $\left(\mathrm{GFP}^{+}\right)$. Dashed circles represent Cdh3-RGCs $\left(\mathrm{GFP}^{+}\right)$that do not express 1264 tdTomato.

1265

1266 (E-F2") Photomicrographs showing the overlap between the projection pattern of 1267 tdTomato ${ }^{+}$RGCs (Cre-DOG targeted) and Cdh3-RGCs. TdTomato ${ }^{+}$RGC axons terminate 1268 in Cdh3-RGC brain targets, the OPN and mdPPN. Dashed white line marks the boundaries 1269 of retinorecipient targets. Arrowheads in close up view (F1, F2) indicate tdTomato ${ }^{+}$axons 1270 innervating the mdPPN.

1271

1272 (G) Targeting of Cdh3-RGCs using Cre-DOG system. AAV viruses encoding split Cre 1273 components (N-Cre and C-Cre) injected binocularly into mice carrying both the Cdh3-GFP 1274 and floxed-Stop hM3D transgenes.

1275

1276 (H) Experimental timeline. 
1278 (I, J) Examples (I) and quantification (J) of pupil size before (-CNO) and after (+CNO)

1279 administration in $\mathrm{Cdh} 3-\mathrm{RGC}$ targeted $\left(\mathrm{Cdh} 3 \mathrm{GFP}^{+} ; \mathrm{hM}^{+} \mathrm{D}^{+}\right.$; $\mathrm{Cre}^{+}$; relative pupil size post-

1280 CNO Mean \pm SEM: $1 \pm 0.03$; $\mathrm{n}=9$ mice $)$ and control mice (Cdh3GFP+; floxed-Stop hM3D;

1281 Cre-; relative-pupil-size post-CNO Mean \pm SEM: $1.1 \pm 0.02$ ).

1282

1283 See also Figure S5.

1284

1285 tdTom: tdTomato reporter; GCL: ganglion cell layer; IPL: inner plexiform layer; INL: 1286 inner nuclear layer; OPN: olivary pretectal nucleus; mdPPN: medial division of the 1287 posterior pretectal nucleus; hM3D: human M3 muscarinic designer receptor; CNO: 1288 Clozapine N-oxide

1289

1290 *** $\mathrm{p}<0.001$, paired Student's t-test. Scale bars: $10 \mu \mathrm{m}$ (D”), $100 \mu \mathrm{m}$ (E”, F”)

1291

1292

1293

1294

1295

1296

1297

1298

1299

1300

1301

1302

1303

1304

1305

1306

1307

1308 
bioRxiv preprint doi: https://doi.org/10.1101/500868; this version posted December 19,2018 . The copyright holder for this preprint (which was

not certified by peer review) is the author/funder, who has granted bioRxiv a license to display the preprint in perpetuity. It is made available

Figurire 51

\section{Tbx20-RGCs (GFP) at different ages}

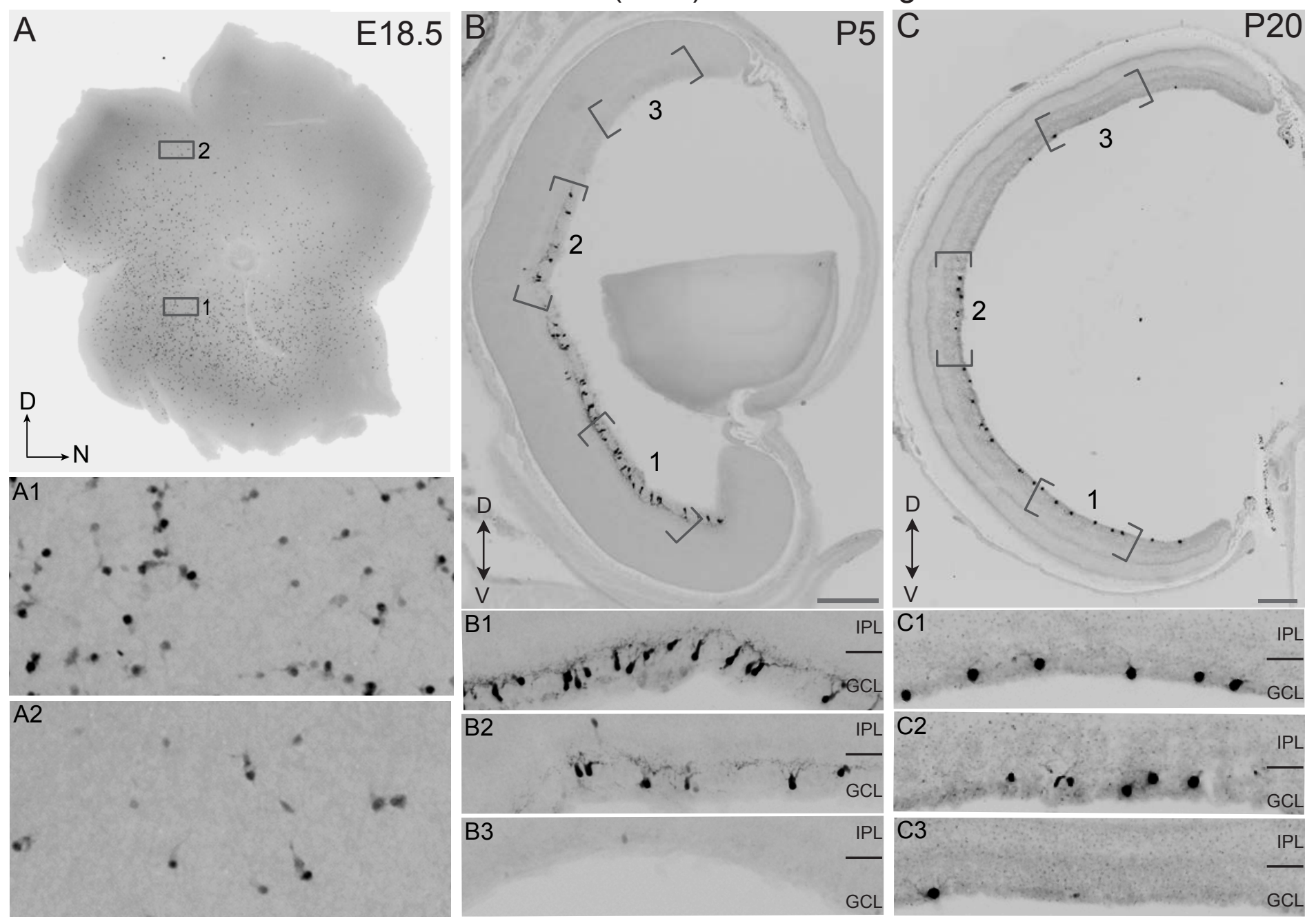




\section{Supplemental Information}

1310

1311 Figure S1, related to Figure 1: Tbx20 is highly enriched in a group of uniquely

\section{2 distributed non-image-forming RGCs that include Cdh3-RGCs}

1313 (A-A2) Flat mount retina showing Tbx20-RGCs (black) are arranged in a ventral/high to 1314 dorsal/low gradient of distribution at E18.5.

1315

1316 (B-C3) Retinal cross-sections showing ventral/high to dorsal/low gradient of distribution 1317 of Tbx20-RGCs (black) at P5 and P20.

1318

1319 D: dorsal; N: nasal; V: ventral; GCL: ganglion cell layer; IPL: inner plexiform layer. Scale 1320 bar: $200 \mu \mathrm{m}(\mathrm{B}, \mathrm{C})$.

1321

1322

1323

1324

1325

1326

1327

1328

1329

1330

1331

1332

1333

1334

1335

1336

1337

1338

1339 
bioRxiv preprint doi: https://doi.org/10.1101/500868; this version posted December 19, 2018. The copyright holder for this preprint (which was not certified by peer review) is the author/funder, who has granted bioRxiv a license to display the preprint in perpetuity. It is made available under aCC-BY-ND 4.0 International license.

\section{Figure S2}

Tbx20-GFP

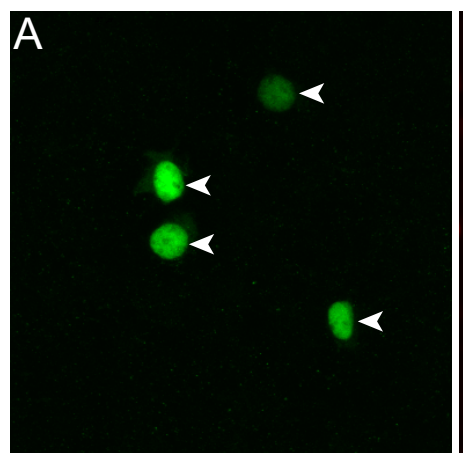

Tbx20-GFP

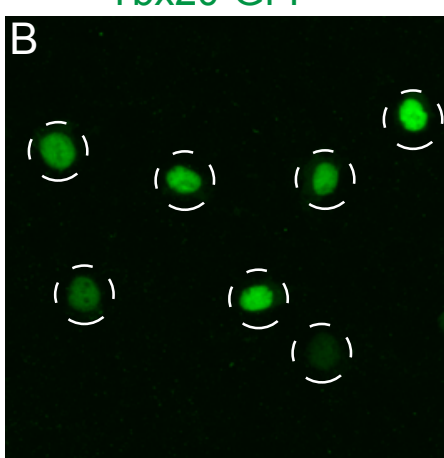

Tbx20-GFP

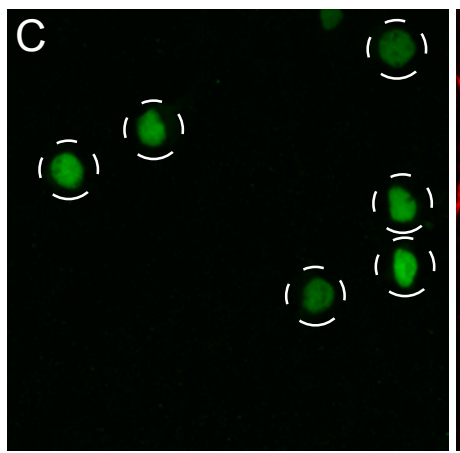

Tbx20-GFP

D

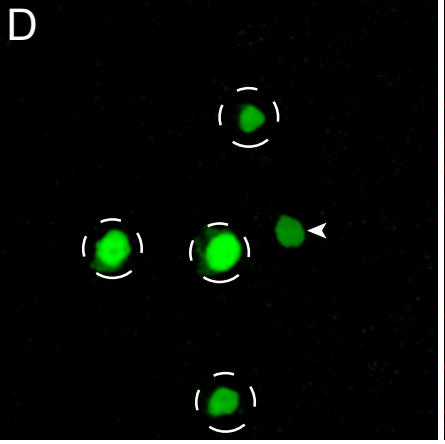

Tbr2

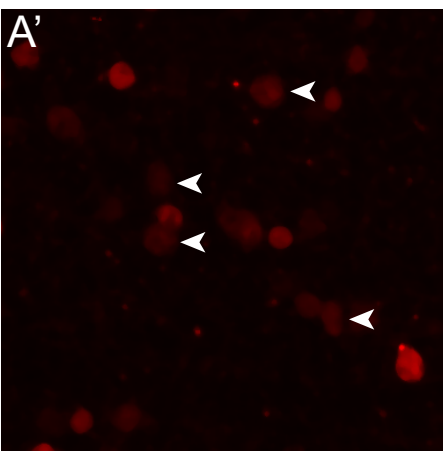

Cart

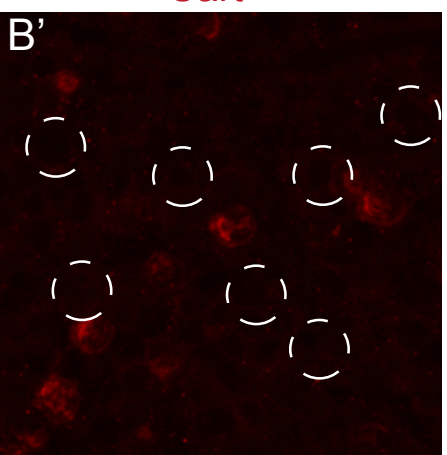

SMI32

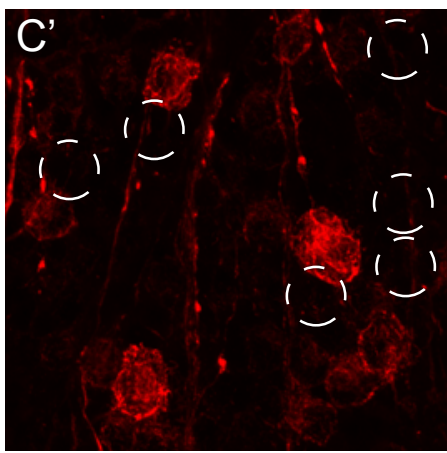

Melanopsin (Mel)

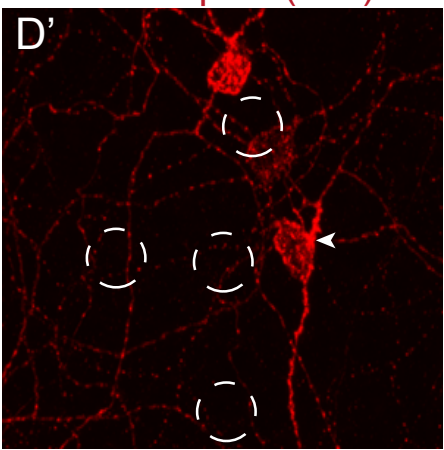

Tbx20-GFP/Tbr2/DAPI

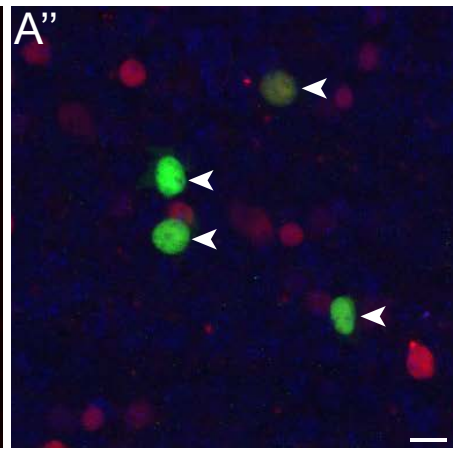

Tbx20-GFP/Cart/DAPI

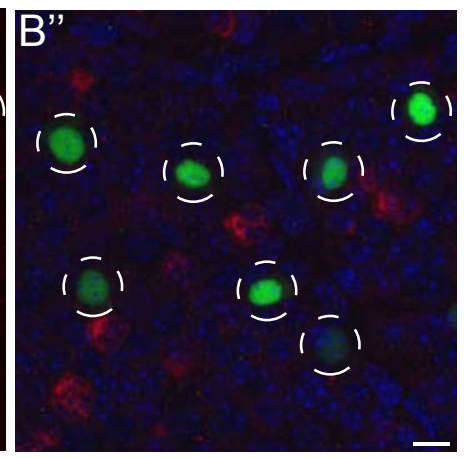

Tbx20-GFP/SMI32/DAPI

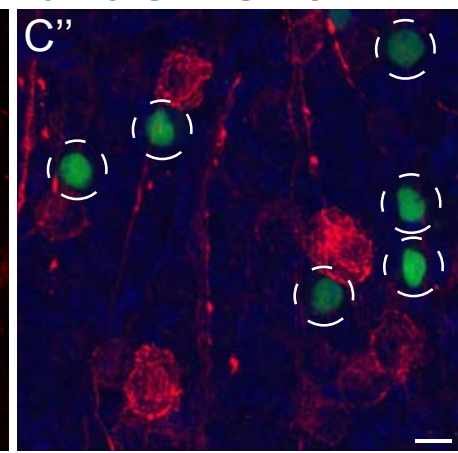

Tbx20-GFP/Mel/DAPI

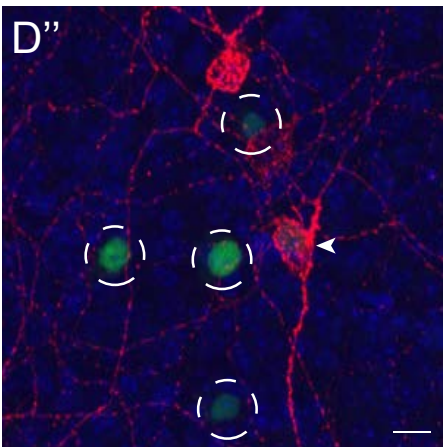


1340 Figure S2, related to Figure 1: RGC type-specific molecular marker analysis suggests 1341 Tbx20-RGCs are part of the non-image-forming RGC family

1342 (A-D”) Photomicrographs showing Tbx20-RGCs (GFP) stained with Tbr2, a marker of 1343 non-image-forming RGCs. Arrowhead indicates overlap of GFP and Tbr2 and open circles 1344 indicate lack thereof.

1345

1346 (B-C”) Photomicrographs showing Tbx20-RGCs (GFP) stained with Cart, a marker for 1347 direction-selective RGCs or SMI-32, a marker for alpha-like RGCs. Open circles indicate 1348 a lack of overlap between markers.

1350 (D-D”) Photomicrographs showing Tbx20-RGCs (GFP) stained with Melanopsin. 1351 Arrowhead indicates overlap of GFP and melanopsin. Open circles indicate a lack of 1352 overlap between these two markers.

1353 Scale bar: $10 \mu \mathrm{m}$ (A", B", C", D").

1354

1355

1356

1357

1358

1359

1360

1361

1362

1363

1364

1365

1366

1367

1368 


\section{Figure S3} under aCC-BY-ND 4.0 International license.
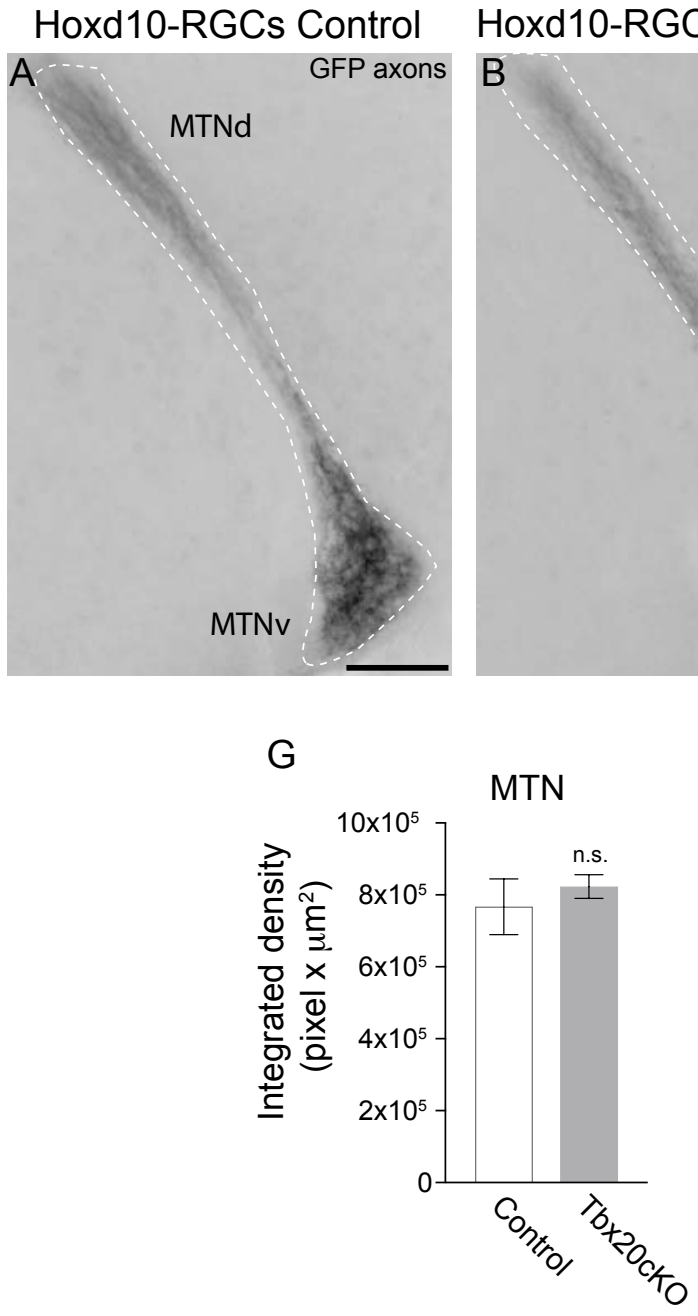
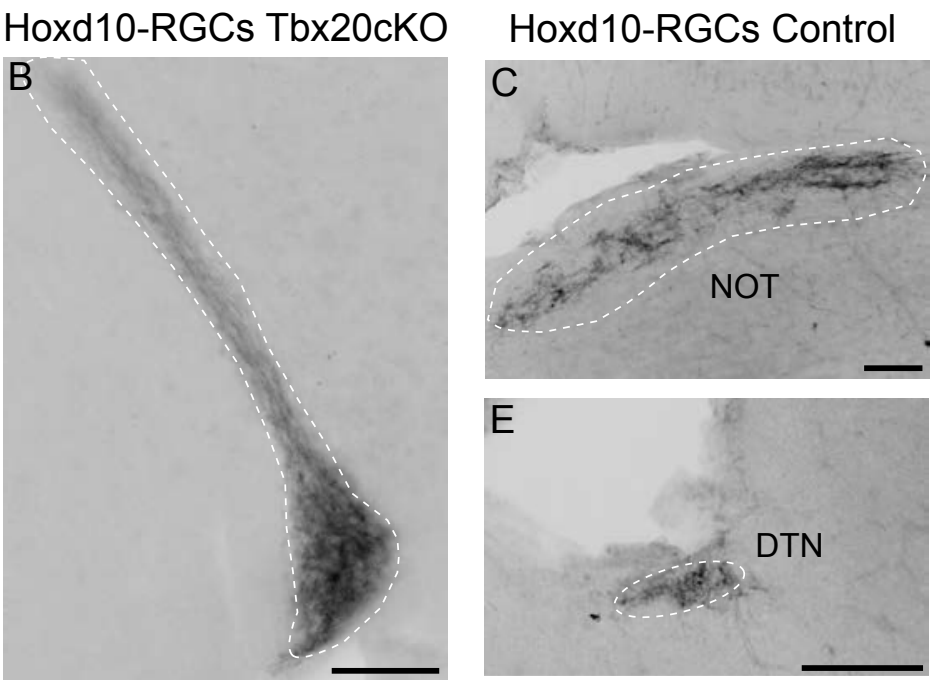

Hoxd10-RGCs Tbx20cKO
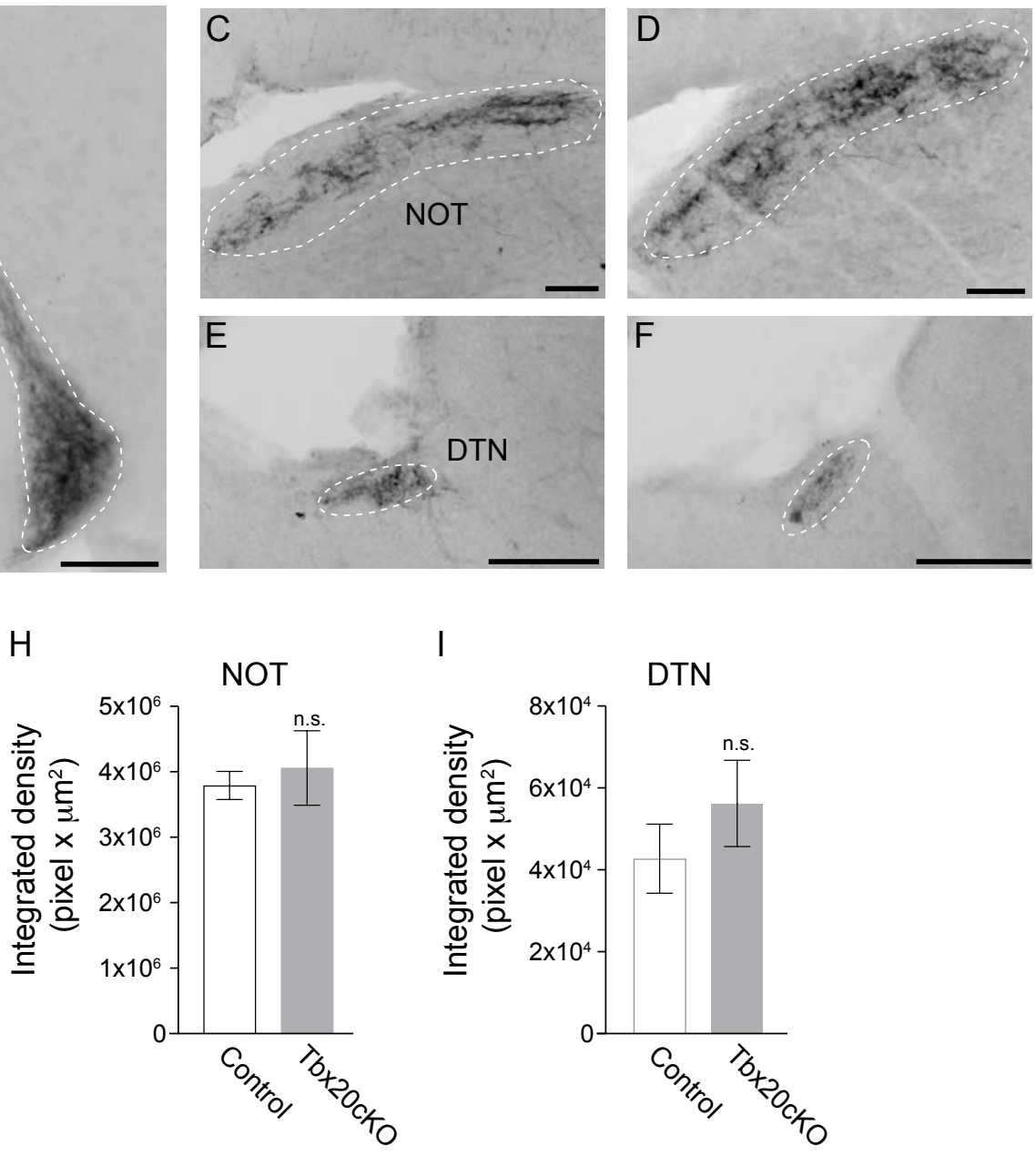

I

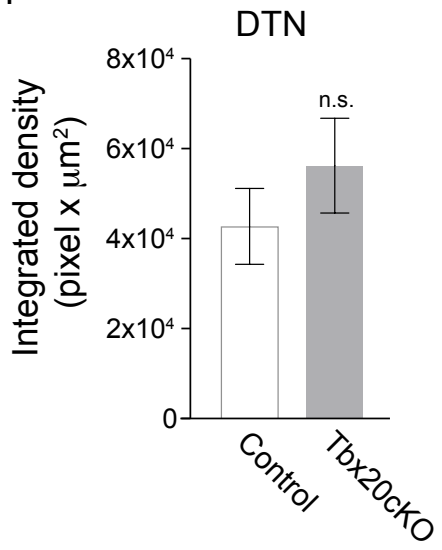


1370 Figure S3, related to Figure 2: Conditional deletion of Tbx20 from accessory optic

1371

1372

1373

1374

1375

1376

1377

1378

1379

1380

1381

1382

1383

1384

1385

1386

1387

1388

1389

1390

1391

1392

1393

1394

1395

1396

1397

1398

1399

\section{system projecting RGCs does not alter their target innervation-density}

(A-F) Photomicrographs showing Hoxd10-RGC axonal projections (GFP, black) to the targets comprising the accessory optic system, MTN, NOT and to the DTN, in a littermate control mouse (A, C, E) and Tbx20 cKO mouse (B, D, F).

(G-I) Quantification of density of Hoxd10-RGC axonal innervation in MTN (G) (Control Mean \pm SEM: $7.7 \times 10^{5} \pm 7.7 \times 10^{4}$ pixel $x \mu \mathrm{m}^{2}$; Tbx $20 \mathrm{cKO}$ Mean \pm SEM: $8.2 \times 10^{5} \pm 3.3 \times 10^{4}$ pixel $x \mu \mathrm{m}^{2}$ ), NOT (I) (Control Mean \pm SEM: $3.8 \times 10^{6} \pm 2.2 \times 10^{5}$ pixel $\times \mu^{2}$; Tbx $20 \mathrm{cKO}$ Mean \pm SEM: $4.1 \times 10^{6} \pm 5.7 \times 10^{5}$ pixel $\times \mu m^{2}$ ) and DTN (I) (Control Mean \pm SEM: 4.3 $\mathrm{x} 10^{4} \pm 8.4 \times 10^{3}$ pixel $\mathrm{x} \mu \mathrm{m}^{2}$; Tbx20 cKO Mean \pm SEM: $5.6 \times 10^{4} \pm 1.1 \times 10^{4}$ pixel $\mathrm{x} \mu \mathrm{m}^{2}$ ) in control ( $\mathrm{n}=5$ mice) and Tbx20 cKOs ( $\mathrm{n}=4$ mice).

MTNd: dorsal medial terminal nucleus; MTNv: ventral MTN; NOT: nucleus of the optic tract; DTN: dorsal terminal nucleus.

n.s.: not significant, $\mathrm{p}>0.05$. Student's t-test. Scale bars: $100 \mu \mathrm{m}$ (A-D), $50 \mu \mathrm{m}$ (E, F). 

Figure 34 peer review) is the author/funder, who has granted bioRxiv a license to displayt

A $\%$ cells expressing Tph2-Cre
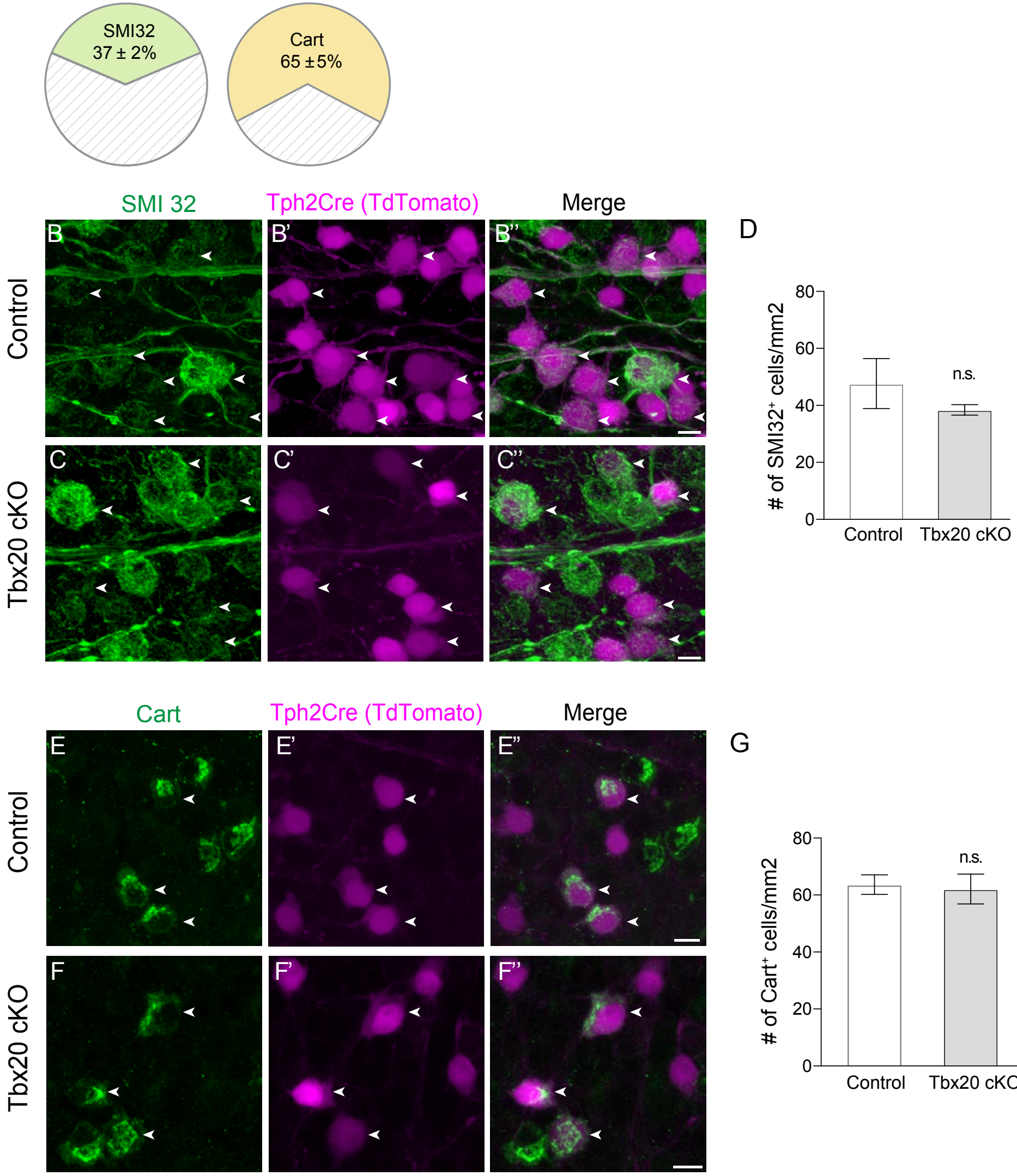

G

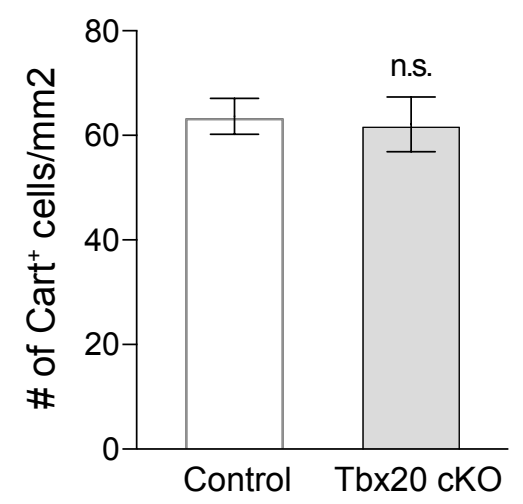


1400 Figure S4, related to Figure 2: Loss of Tbx20 in other RGC subtypes does not alter 1401 their density

1402 (A) Quantification of SMI-32+ cells that express Tph2-Cre ( $\mathrm{n}=3$ retinas/mice; 144 SMI32 ${ }^{+}$

1403 cells) and Cart ${ }^{+}$cells that express Tph2-Cre ( $n=5$ retinas/mice; 321 Cart $^{+}$cells $)$at P25. Data

1404 shown are Mean \pm SEM.

1405

1406 (B-C”) Photomicrographs showing SMI32 and Tph2-Cre (tdTomato) expression in control 1407 and Tbx20 cKO mice. White arrowheads indicate co-expression.

1408 (D) Quantification of density of SMI32+ RGCs in control and Tbx20 cKO mice (Control 1409 Mean \pm SEM: $48 \pm 9$ cells $/ \mu \mathrm{m}^{2}, \mathrm{n}=3$ mice; Tbx $20 \mathrm{cKO}$ Mean \pm SEM: $39 \pm 2$ cells $/ \mu \mathrm{m}^{2}$, $1410 \mathrm{n}=3$ mice).

1411

1412 (E-F") Photomicrographs showing Cart and Tph2-Cre (tdTomato) expression in control 1413 and Tbx20 cKO mice. White arrowheads indicate co-expression.

1414

1415 (G) Quantification of density of Cart+ RGCs in control and Tbx20 cKO mice (Control 1416 Mean \pm SEM: $64 \pm 3$ cells $/ \mu \mathrm{m}^{2}, \mathrm{n}=5$ mice; Tbx20 cKO Mean \pm SEM: $63 \pm 5$ cells $/ \mu \mathrm{m}^{2}$, $1417 \mathrm{n}=3$ mice).

1418 n.s.: (not significant): p>0.05. Student's t-test. Scale bar: $10 \mu \mathrm{m}$ (B”, C”, E”, F”) 
bioRxiv preprint doi: https://doi.org/10.1101/500868; this version posted December 19,2018 . The copyright holder for this preprint (which was not certified by peer review) is the author/funder, who has granted bioRxiv a license to display the preprint in perpetuity. It is made available under ACC-BY-ND 4.0 International license.

\section{Figure S5}

A

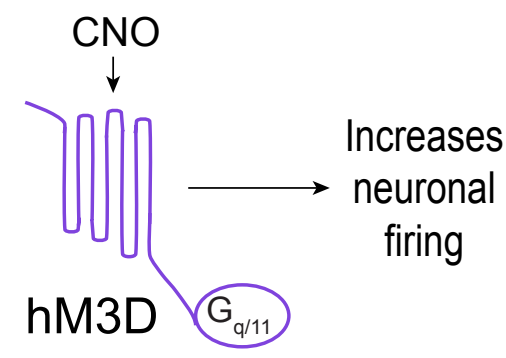

C

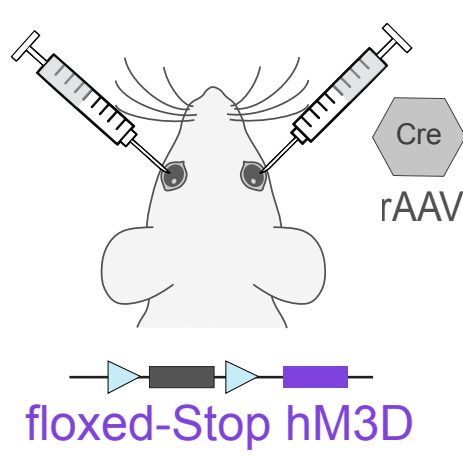

B
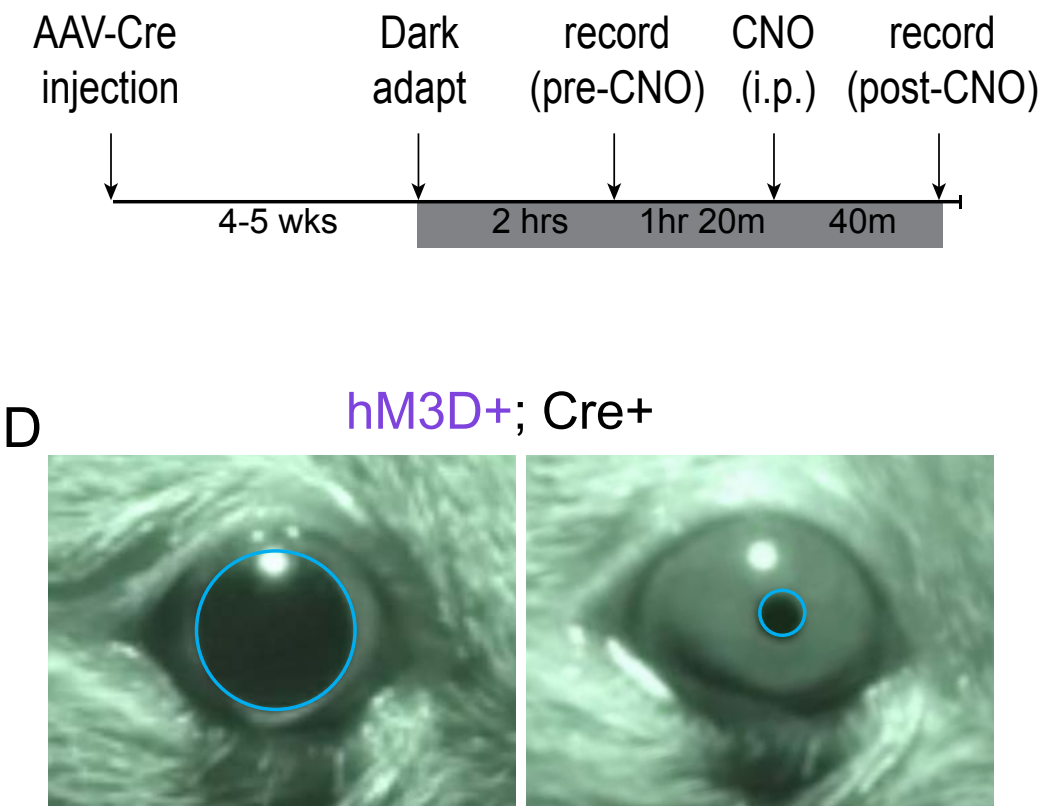

$-\mathrm{CNO}$

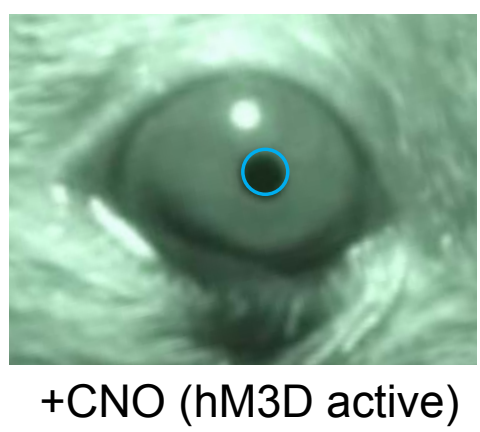




\section{Figure S5, related to Figure 7: Chemogenetic activation of RGCs generates robust}

\section{2 pupil constriction.}

1433 (A) Schematic of Gq coupled hM3D receptor.

1434

1435 (B, C) Experimental timeline (B). AAVs encoding Cre recombinase were injected into both 1436 eyes of floxed-STOP hM3D mice (C). 4-5 weeks post-injection pupil size was measured 1437 after dark adaptation before and after intraperitoneal (i.p.) CNO (Clozapine N-oxide) 1438 administered (B).

1440 (D) Example of pupil size pre (left) and post (right) CNO administration. 